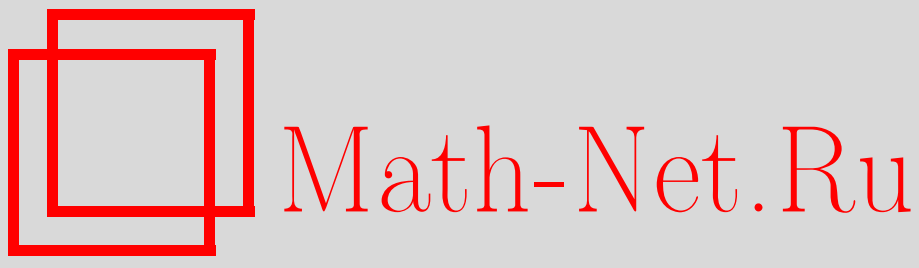

Г. С. Маканин, Конечная параметризация решений уравнений в свободном моноиде. II, Матем. сб., 2004, том 195, номер 4, 65-96

DOI: https://doi.org/10.4213/sm814

Использование Общероссийского математического портала Math-Net.Ru подразумевает, что вы прочитали и согласны с пользовательским соглашением

http://www . mathnet.ru/rus/agreement

Параметры загрузки:

IP: 3.85 .5 .30

26 апреля 2023 г., $16: 20: 25$ 
УДК 519.4

\author{
Г.С. Маканин
}

\title{
Конечная параметризация решений уравнений в свободном моноиде. II
}

В предыдущей работе автора были введены параметризующие функции $\mathbf{F i}$, Th, Ro, зависящие от словарных переменных, натуральных переменных и переменных, значениями которых являются конечные последовательности натуральных переменных. С помощью параметризующих функций $\mathbf{F i}, \mathbf{T h}, \mathbf{R o}$ выписываются конечные формулы для семейства решений всякого уравнения вида $\varphi\left(x_{1}, x_{2}, x_{3}\right) x_{4}=\psi\left(x_{1}, x_{2}, x_{3}\right) x_{5}$, где $\varphi\left(x_{1}, x_{2}, x_{3}\right), \psi\left(x_{1}, x_{2}, x_{3}\right)$ - произвольные слова в алфавите $x_{1}, x_{2}, x_{3}$ в свободном моноиде.

Библиография: 12 названий.

\section{§1. Введение}

Параметризовать решения уравнения в свободном моноиде - это значит выписать общее решение (множество всех решений) этого уравнения при помоши конечных формул, зависящих от параметров. В 1967 году Хмелевский [1] показал, что обшее решение всякого уравнения с тремя неизвестными в свободном моноиде может быть параметризовано конечньм числом формул, зависящих от переменных двух типов: словарных переменных и натуральных переменных. Такая параметризация называется примитивной. В 1971 году Хмелевский [2] доказал, что общее решение уравнения Маркова

$$
x_{1} x_{2} x_{3}=x_{3} x_{1} x_{4}
$$

примитивной параметризации не допускает.

Чтобы преодолеть возникший барьер и расширить класс параметризуемых уравнений, нужно было изыскивать новые возможности, создавать новый метод параметризации решений уравнений. Такой метод был найден, разработан и использован в работах [3]-[11]. Поворотньм пунктом в создании новой параметризации явилось понимание необходимости введения переменной третьего типа, а именно переменной, значениями которой являются конечные последовательности натуральных переменных. Вместе с открытием новой переменной возникло понятие параметризующей функции, зависящей от переменных всех трех типов. Параметризующая функция - это рекурсивная функция, рекурсия которой конструируется при помощи последовательности натуральных переменных.

Главным результатом настоящей работы являются выписанные конечные формулы для параметризации решений всякого уравнения из класса уравнений вида

$$
\varphi\left(x_{1}, x_{2}, x_{3}\right) x_{4}=\psi\left(x_{1}, x_{2}, x_{3}\right) x_{5},
$$

Работа вьполнена при поддержке Российского фонда фундаментальных исследований. 
где $\varphi\left(x_{1}, x_{2}, x_{3}\right), \psi\left(x_{1}, x_{2}, x_{3}\right)$ - произвольные слова в алфавите $x_{1}, x_{2}, x_{3}$ в свободном моноиде. Вводятся параметризующие функции

$$
\begin{aligned}
& \mathbf{F i}_{\left(x_{1}, x_{2}\right)^{\lambda_{1}, \ldots, \lambda_{s}}, \quad s \geqslant 0,} \\
& \mathbf{T h}_{\left(x_{1}, x_{2}, x_{3}\right)_{i}^{\lambda_{1}, \ldots, \lambda_{2 s}}, \quad i=1,2,3, s \geqslant 0,}
\end{aligned}
$$

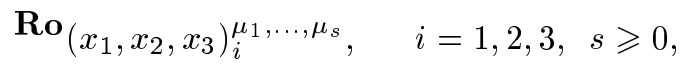

зависящие от словарных переменных $x_{1}, x_{2}, x_{3}$, натуральных переменных $\lambda_{k}$ и переменных $\mu_{k}$, значениями которых являются последовательности натуральных переменных. Суперпозиции этих функций и примитивно параметризуемых слов конструируют формулы для параметризации решений уравнений из класса уравнений

$$
\varphi\left(x_{1}, x_{2}, x_{3}\right) x_{4}=\psi\left(x_{1}, x_{2}, x_{3}\right) x_{5} .
$$

Указанный класс уравнений является максимальным классом, уравнения которого можно параметризовать при помощи известных параметризующих функций (см. [6]-[11]). Он включает в себя все классы на сегодня уже параметризованных уравнений (за исключением некоторых квадратичных уравнений) и большое количество известных уже уравнений, ранее не поддававшихся параметризации.

Настоящая работа является продолжением работы [12], тем самым нумерация утверждений и уравнений сохраняется.

\section{§2. Вырожденные уравнения}

Произведение параметрических преобразований, состоящее из примитивных параметрических преобразований и не более трех преобразований функцией $\mathbf{F i}$, будем называть тройным $\mathbf{F i}_{-}$преобразованием. Результат применения тройного $\mathbf{F i}$-преобразования к равенству слов в алфавите словарных переменных будем на-

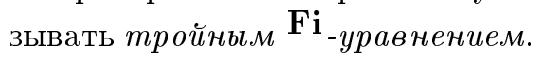

Все уравнения, которые мы рассматриваем в этой части, являются тройными $\mathbf{F i}$-уравнениями.

Иногда утверждение формулируется в сокращенной записи: выписываются только уравнения и номера утверждений, к которьм оно сводится. Преобразования легко восстанавливаются по виду префикса уравнения.

УТВЕРЖДЕНИЕ 64. Общее решение уравнения

$$
x_{1}^{\alpha+1} x_{2} P\left(x_{1}, x_{2}, \lambda_{1}, \ldots, \lambda_{r}\right) x_{3}=x_{2}^{\beta+1} x_{1} Q\left(x_{1}, x_{2}, \lambda_{1}, \ldots, \lambda_{r}\right) x_{4}
$$

где $\alpha, \beta, \lambda_{1}, \ldots, \lambda_{r}$ - натуральные параметры, описывается преобразования$\mathcal{M u}$

$$
\left\{\begin{array} { l } 
{ x _ { 1 } \rightarrow x _ { 1 } ^ { \eta } , } \\
{ x _ { 2 } \rightarrow x _ { 1 } ^ { \theta } , } \\
{ x _ { 3 } \rightarrow x _ { 1 } ^ { k } x _ { 4 } , }
\end{array} \quad \left\{\begin{array}{l}
x_{1} \rightarrow x_{1}^{\sigma}, \\
x_{2} \rightarrow x_{1}^{\tau}, \\
x_{4} \rightarrow x_{1}^{\xi} x_{3},
\end{array}\right.\right.
$$

әде $\eta, \theta, k, \sigma, \tau, \xi$ - натуральные параметры с наследованными из уравнения условиями. 
ДокАЗАТЕЛЬСТво непосредственно следует из утверждения 31.

УТВЕРЖДЕНИЕ 65. Общее решение уравнения

$$
x_{1}^{\alpha+1} x_{2} P\left(x_{1}, x_{2}, \lambda_{1}, \ldots, \lambda_{r}\right) x_{3}=x_{2}^{\beta+1} x_{4},
$$

где $\alpha, \beta, \lambda_{1}, \ldots, \lambda_{r}$ - натуральные параметры, описьвается следующими шестью преобразованиями:

$$
\begin{aligned}
& \left\{\begin{array} { l } 
{ x _ { 1 } \rightarrow x _ { 1 } ^ { \eta } , } \\
{ x _ { 2 } \rightarrow x _ { 1 } ^ { \theta } } \\
{ x _ { 3 } \rightarrow x _ { 1 } ^ { k } x _ { 4 } }
\end{array} \quad \left\{\begin{array}{l}
x_{1} \rightarrow x_{1}^{\sigma}, \\
x_{2} \rightarrow x_{1}^{\tau} \\
x_{4} \rightarrow x_{1}^{\xi} x_{3}
\end{array}\right.\right. \\
& \left\{\begin{array}{l}
x_{1} \rightarrow x_{2}^{\beta+1} x_{1} \\
x_{4} \rightarrow x_{1}\left(x_{2}^{\beta+1} x_{1}\right)^{\alpha} x_{2} P x_{3} ;
\end{array}\right. \\
& \left\{\begin{array}{l}
\beta \rightarrow 0 \\
x_{1} \rightarrow x_{2} x_{1} \\
x_{2} \rightarrow\left(x_{2} x_{1}\right)^{\lambda} x_{2} \\
x_{4} \rightarrow\left(x_{1} x_{2}\right)^{\alpha+1} P x_{3}
\end{array}\right. \\
& \left\{\begin{array}{l}
\alpha \rightarrow 0 \\
\beta \rightarrow \delta+1 \\
x_{1} \rightarrow\left(\left(x_{2} x_{1}\right)^{\lambda} x_{2}\right)^{\delta+1} x_{2} x_{1} \\
x_{2} \rightarrow\left(x_{2} x_{1}\right)^{\lambda} x_{2} \\
x_{4} \rightarrow x_{1} x_{2} P x_{3}
\end{array}\right. \\
& \left\{\begin{array}{l}
\alpha \rightarrow \gamma+1 \\
\beta \rightarrow \delta+1 \\
x_{1} \rightarrow x_{2} x_{1} \\
x_{2} \rightarrow\left(x_{2} x_{1}\right)^{\lambda} x_{2} \\
x_{4} \rightarrow x_{1} x_{2}\left(\left(x_{2} x_{1}\right)^{\lambda+1} x_{2}\right)^{\delta} x_{2} x_{1}\left(\left(\left(x_{2} x_{1}\right)^{\lambda+1} x_{2}\right)^{\delta+1} x_{2} x_{1}\right)^{\gamma}\left(x_{2} x_{1}\right)^{\lambda+1} x_{2} x_{3}
\end{array}\right.
\end{aligned}
$$

где $\eta, \theta, k, \lambda, \gamma, \delta, \sigma, \tau, \xi$ - натуральные параметры с наследованныцми из уравнения условиями.

ДокАЗАТЕЛЬСтво непосредственно следует из утверждения 30 .

УТВЕРЖДЕНИЕ 66. Общее решение уравнения

$$
P\left(x_{1}, x_{2}, \lambda_{1}, \ldots, \lambda_{r}\right) x_{3}=x_{4},
$$

где $\alpha, \beta, \lambda_{1}, \ldots, \lambda_{r}$ - натуральные параметры, описывается преобразованием

$$
x_{4} \rightarrow P\left(x_{1}, x_{2}, \lambda_{1}, \ldots, \lambda_{r}\right) x_{3} .
$$

ДоКАЗАТЕЛЬСТво очевидно.

УТВЕРЖ ДЕНИЕ 67. Параметрическое уравнение

$$
P\left(x_{1}, x_{2}, \lambda_{1}, \ldots, \lambda_{r}\right) x_{3}=Q\left(x_{1}, x_{2}, \lambda_{1}, \ldots, \lambda_{r}\right) x_{4},
$$

где $\lambda_{1}, \ldots, \lambda_{r}$ - натуральные параметры, разделяется в список уравнений вида (64), (65), (29), (66).

ДоказАТЕЛЬСтво следует из теорем Fi3-Fi5. 


\section{§3. Тройные $\mathrm{Fi}_{\text {-уравнения }}$}

Все уравнения,которые мы рассматриваем в этом параграфе, являются тройными $\mathbf{F i}$-уравнениями.

УТВЕРЖДЕНИЕ 68. Общее решение уравнения

$$
x_{3} x_{2} x_{1} x_{3} P\left(x_{1}, x_{2}, x_{3}, \lambda_{1}, \ldots, \lambda_{r}\right) x_{4}=x_{1} x_{2} x_{1} x_{3} Q\left(x_{1}, x_{2}, x_{3}, \lambda_{1}, \ldots, \lambda_{r}\right) x_{5},
$$

где $\alpha, \lambda_{1}, \ldots, \lambda_{r}$ - натуральные параметрь, описывается преобразованиями

$$
x_{3} \rightarrow x_{1} x_{3}, \quad x_{1} \rightarrow x_{3} x_{1}
$$
$\langle 13\rangle$,
$\langle 67\rangle$;
$\langle 67\rangle$.

УТВЕРЖДЕНИЕ 69. Общее решение уравнения

$x_{3} x_{2} x_{1} x_{2} x_{3} P\left(x_{1}, x_{2}, x_{3}, \lambda_{1}, \ldots, \lambda_{r}\right) x_{4}=x_{1}^{2} x_{2} x_{3} x_{2} Q\left(x_{1}, x_{2}, x_{3}, \lambda_{1}, \ldots, \lambda_{r}\right) x_{5},(69)$ где $\alpha, \lambda_{1}, \ldots, \lambda_{r}$ - натуральные параметры, описывается преобразованиями $x_{3} \rightarrow x_{1} x_{3}, \quad x_{1} \rightarrow x_{3} x_{1}$,

УТВЕРЖДДЕНИЕ 70. Общее решение уравнения

$$
x_{3} x_{2} x_{1} x_{3} P\left(x_{1}, x_{2}, x_{3}, \lambda_{1}, \ldots, \lambda_{r}\right) x_{4}=x_{2} x_{1}^{2} x_{3}^{2} Q\left(x_{1}, x_{2}, x_{3}, \lambda_{1}, \ldots, \lambda_{r}\right) x_{5},
$$

где $\lambda_{1}, \ldots, \lambda_{r}$ - натуральнье параметры, описывается преобразованиями

$$
\begin{array}{ll}
x_{2} \rightarrow x_{3}^{\alpha} x_{2}, & x_{3} \rightarrow 1, \\
x_{3} \rightarrow x_{2} x_{3}, & \langle 67\rangle,
\end{array}
$$

әде $\alpha$ - натуральный параметр.

УТВЕРЖДЕНИЕ 71. Общее решение уравнения

$$
x_{3} x_{2} x_{1} x_{3} P\left(x_{1}, x_{2}, x_{3}, \lambda_{1}, \ldots, \lambda_{r}\right) x_{4}=x_{2} x_{1}^{2} x_{3} x_{2} Q\left(x_{1}, x_{2}, x_{3}, \lambda_{1}, \ldots, \lambda_{r}\right) x_{5},
$$

где $\lambda_{1}, \ldots, \lambda_{r}$ - натуральные параметры, описывается преобразованиями

$$
\begin{array}{ll}
x_{2} \rightarrow x_{3}^{\alpha} x_{2}, & x_{3} \rightarrow 1, \\
x_{3} \rightarrow x_{2} x_{3}, & \langle 67\rangle,
\end{array}
$$$$
\langle 69\rangle
$$

әде $\alpha$ - натуральный параметр. 
УТВЕРЖДЕНИЕ 72. Общее решение уравнения

$x_{3} x_{2} x_{1} x_{2} P\left(x_{1}, x_{2}, x_{3}, \lambda_{1}, \ldots, \lambda_{r}\right) x_{4}=x_{1}\left(x_{2} x_{1}\right)^{\alpha+1} x_{3} x_{2} Q\left(x_{1}, x_{2}, x_{3}, \lambda_{1}, \ldots, \lambda_{r}\right) x_{5}$,

где $\alpha, \lambda_{1}, \ldots, \lambda_{r}$ - натуральнье параметрьи, сводится $\kappa\langle 4\rangle,\langle 67\rangle,\langle 68\rangle,\langle 71\rangle$.

УТВЕРЖДЕНИЕ 73. Общее решение уравнения

$x_{3} x_{2} x_{1}^{2} P\left(x_{1}, x_{2}, x_{3}, \lambda_{1}, \ldots, \lambda_{r}\right) x_{4}=\left(x_{2} x_{1}\right)^{\alpha+1} x_{1} x_{3} x_{2} Q\left(x_{1}, x_{2}, x_{3}, \lambda_{1}, \ldots, \lambda_{r}\right) x_{5}$

әде $\alpha, \lambda_{1}, \ldots, \lambda_{r}$ - натуральные параметрви, сводится $\kappa\langle 4\rangle,\langle 13\rangle,\langle 67\rangle,\langle 68\rangle$.

УТВЕРЖДЕНИЕ 74. Общее решение уравнения

$x_{3} x_{2} x_{1} x_{3} P\left(x_{1}, x_{2}, x_{3}, \lambda_{1}, \ldots, \lambda_{r}\right) x_{4}=\left(x_{2} x_{1}\right)^{\alpha+1} x_{1} x_{3} x_{2} Q\left(x_{1}, x_{2}, x_{3}, \lambda_{1}, \ldots, \lambda_{r}\right) x_{5}$,

әде $\alpha, \lambda_{1}, \ldots, \lambda_{r}$-натуральные параметры, сводится $\kappa\langle 4\rangle,\langle 67\rangle,\langle 68\rangle,\langle 70\rangle,\langle 71\rangle$.

УТВЕРЖДЕНИЕ 75. Общее решение уравнения

$$
\begin{aligned}
& x_{3} x_{2} x_{1} x_{3} P\left(x_{1}, x_{2}, x_{3}, \lambda_{1}, \ldots, \lambda_{r}\right) x_{4} \\
& \quad=\left(\left(x_{1} x_{2}\right)^{\alpha+1} x_{1}\right)^{\delta+1} x_{2} x_{1} x_{3} Q\left(x_{1}, x_{2}, x_{3}, \lambda_{1}, \ldots, \lambda_{r}\right) x_{5},
\end{aligned}
$$

где $\alpha, \delta, \lambda_{1}, \ldots, \lambda_{r}$ - натуральные параметры, сводится $\kappa\langle 4\rangle,\langle 13\rangle,\langle 67\rangle$, $\langle 68\rangle,\langle 72\rangle$.

УТВЕРЖДЕНИЕ 76. Общее решение уравнения

$x_{3} x_{2} x_{1} x_{3} P\left(x_{1}, x_{2}, x_{3}, \lambda_{1}, \ldots, \lambda_{r}\right) x_{4}=x_{1}^{\alpha+1} x_{2} x_{1} x_{3} Q\left(x_{1}, x_{2}, x_{3}, \lambda_{1}, \ldots, \lambda_{r}\right) x_{5}$,

әде $\alpha, \lambda_{1}, \ldots, \lambda_{r}$ - натуральные параметрви, сводится $\kappa\langle 67\rangle,\langle 72\rangle,\langle 4\rangle,\langle 13\rangle$.

УТВЕРЖДЕНИЕ 77. Общее решение уравнения

$$
x_{3} x_{2} x_{1} x_{2} x_{3} P\left(x_{1}, x_{2}, x_{3}, \lambda_{1}, \ldots, \lambda_{r}\right) x_{4}=x_{2}^{\alpha+1} x_{1} x_{2} x_{3} Q\left(x_{1}, x_{2}, x_{3}, \lambda_{1}, \ldots, \lambda_{r}\right) x_{5}
$$

где $\alpha, \lambda_{1}, \ldots, \lambda_{r}$ - натуральнье параметрыл, сводится $\kappa\langle 1\rangle,\langle 4\rangle,\langle 25\rangle,\langle 27\rangle,\langle 67\rangle$.

УТВЕРЖДЕНИЕ 78. Общее решение уравнения

$$
x_{3} x_{2} x_{1} x_{3} P\left(x_{1}, x_{2}, x_{3}, \lambda_{1}, \ldots, \lambda_{r}\right) x_{4}=x_{2}^{2} x_{1} x_{3} Q\left(x_{1}, x_{2}, x_{3}, \lambda_{1}, \ldots, \lambda_{r}\right) x_{5},
$$

әде $\lambda_{1}, \ldots, \lambda_{r}$ - натуральнье параметрьи, сводится $\kappa\langle 1\rangle,\langle 25\rangle,\langle 67\rangle$.

УТВЕРЖДЕНИЕ 79. Общее решение уравнения

$$
x_{3} x_{2} x_{1} x_{2} P\left(x_{1}, x_{2}, x_{3}, \lambda_{1}, \ldots, \lambda_{r}\right) x_{4}=\left(x_{2} x_{1}\right)^{\alpha+2} x_{2} x_{3} Q\left(x_{1}, x_{2}, x_{3}, \lambda_{1}, \ldots, \lambda_{r}\right) x_{5},
$$

где $\alpha, \lambda_{1}, \ldots, \lambda_{r}$ - натуральные параметры, сводится $\kappa\langle 1\rangle,\langle 4\rangle,\langle 9\rangle,\langle 67\rangle$. 
УТВЕРЖДЕНИЕ 80. Общее решение уравнения

$x_{3} x_{2} x_{3} x_{1} x_{2} P\left(x_{1}, x_{2}, x_{3}, \lambda_{1}, \ldots, \lambda_{r}\right) x_{4}=x_{1} x_{2}^{2} x_{3} x_{1} Q\left(x_{1}, x_{2}, x_{3}, \lambda_{1}, \ldots, \lambda_{r}\right) x_{5},(80)$

где $\lambda_{1}, \ldots, \lambda_{r}$ - натуральные параметрьи, сводится $\kappa\langle 8\rangle,\langle 11\rangle,\langle 67\rangle$.

УТВЕРЖДЕНИЕ 81. Общее решение уравнения

$x_{3} x_{1} x_{3} x_{2} x_{1} P\left(x_{1}, x_{2}, x_{3}, \lambda_{1}, \ldots, \lambda_{r}\right) x_{4}=x_{2}\left(x_{2} x_{1}\right)^{\alpha+1} x_{3} Q\left(x_{1}, x_{2}, x_{3}, \lambda_{1}, \ldots, \lambda_{r}\right) x_{5}$,

əде $\alpha, \lambda_{1}, \ldots, \lambda_{r}$ - натуральнье параметры, сводится $\kappa\langle 68\rangle,\langle 75\rangle,\langle 80\rangle$.

УТВЕРЖДЕНИЕ 82. Общее решение уравнения

$$
\begin{aligned}
& x_{3} x_{2} x_{1} x_{2} P\left(x_{1}, x_{2}, x_{3}, \lambda_{1}, \ldots, \lambda_{r}\right) x_{4} \\
& \quad=x_{1} x_{2}\left(x_{2} x_{1}\right)^{\alpha+1} x_{2} x_{3} Q\left(x_{1}, x_{2}, x_{3}, \lambda_{1}, \ldots, \lambda_{r}\right) x_{5},
\end{aligned}
$$

где $\alpha, \lambda_{1}, \ldots, \lambda_{r}$ - натуральные параметры, сводится $\kappa\langle 1\rangle,\langle 9\rangle,\langle 67\rangle,\langle 79\rangle$.

УТВЕРЖДЕНИЕ 83. Общее решение уравнения

$$
x_{3} x_{2} x_{1} x_{2} P\left(x_{1}, x_{2}, x_{3}, \lambda_{1}, \ldots, \lambda_{r}\right) x_{4}=x_{1}^{2} x_{2} x_{3} x_{2} Q\left(x_{1}, x_{2}, x_{3}, \lambda_{1}, \ldots, \lambda_{r}\right) x_{5}
$$

әде $\lambda_{1}, \ldots, \lambda_{r}$ - натуральные параметры, описъьвается преобразованиями

$$
x_{1} \rightarrow x_{3} x_{1}, \quad x_{3} \rightarrow x_{1} x_{3}
$$

УТВЕРЖДЕНИЕ 84. Общее решение уравнения

$$
x_{3} x_{2} x_{1} x_{2} P\left(x_{1}, x_{2}, x_{3}, \lambda_{1}, \ldots, \lambda_{r}\right) x_{4}=x_{2} x_{1}^{2} x_{3}^{2} Q\left(x_{1}, x_{2}, x_{3}, \lambda_{1}, \ldots, \lambda_{r}\right) x_{5},
$$

где $\lambda_{1}, \ldots, \lambda_{r}$ - натуральные параметры, описывается преобразованиями

$$
x_{3} \rightarrow x_{2} x_{3}, \quad x_{2} \rightarrow x_{3} x_{2}
$$

УТВЕРЖДЕНИЕ 85. Общее решение уравнения

$$
x_{3} x_{2} x_{1} x_{2} P\left(x_{1}, x_{2}, x_{3}, \lambda_{1}, \ldots, \lambda_{r}\right) x_{4}=x_{2} x_{1}^{2} x_{3} x_{2} Q\left(x_{1}, x_{2}, x_{3}, \lambda_{1}, \ldots, \lambda_{r}\right) x_{5}
$$

где $\lambda_{1}, \ldots, \lambda_{r}$ - натуральные параметрь, описывается преобразованиями

$$
x_{3} \rightarrow x_{2} x_{3}, \quad x_{2} \rightarrow x_{3} x_{2}
$$


УТВЕРЖДЕНИЕ 86. Общее решение уравнения

$$
x_{3} x_{2} x_{1} x_{3} P\left(x_{1}, x_{2}, x_{3}, \lambda_{1}, \ldots, \lambda_{r}\right) x_{4}=x_{1}^{2} x_{2} x_{3} x_{2} Q\left(x_{1}, x_{2}, x_{3}, \lambda_{1}, \ldots, \lambda_{r}\right) x_{5},
$$

где $\lambda_{1}, \ldots, \lambda_{r}$ - натуральные параметрь, описъьвается преобразованиями

$$
x_{3} \rightarrow x_{1} x_{3}, \quad x_{1} \rightarrow x_{3} x_{1},
$$

$\langle 67\rangle$;

УТВЕРЖДЕНИЕ 87. Общее решение уравнения

$x_{2} x_{1} x_{3} x_{1} P\left(x_{1}, x_{2}, x_{3}, \lambda_{1}, \ldots, \lambda_{r}\right) x_{4}=x_{3}\left(x_{1} x_{3}\right)^{\alpha+1} x_{2} x_{1} Q\left(x_{1}, x_{2}, x_{3}, \lambda_{1}, \ldots, \lambda_{r}\right) x_{5}$,

где $\alpha, \lambda_{1}, \ldots, \lambda_{r}$-натуральные параметры, сводится $\kappa\langle 4\rangle,\langle 13\rangle,\langle 67\rangle,\langle 70\rangle,\langle 71\rangle$.

УТВЕРЖДЕНИЕ 88. Общее решение уравнения

$x_{2} x_{1} x_{3} x_{i} P\left(x_{1}, x_{2}, x_{3}, \lambda_{1}, \ldots, \lambda_{r}\right) x_{4}=x_{3}\left(x_{1} x_{3}\right)^{\alpha+1} x_{2} x_{1} Q\left(x_{1}, x_{2}, x_{3}, \lambda_{1}, \ldots, \lambda_{r}\right) x_{5}$,

где $i=2,3, \alpha, \lambda_{1}, \ldots, \lambda_{r}$ - натуральнье параметры, сводится $\kappa\langle 4\rangle,\langle 13\rangle$, $\langle 67\rangle,\langle 85\rangle$.

\section{§4. Циклические решения}

Мы будем говорить, что общее решение тройного $\mathbf{F i}$-уравнения

$$
P\left(x_{1}, x_{2}, x_{3}, \lambda_{1}, \ldots, \lambda_{r}, \mu_{1}, \mu_{2}, \mu_{3}\right) x_{4}=Q\left(x_{1}, x_{2}, x_{3}, \lambda_{1}, \ldots, \lambda_{r}, \mu_{1}, \mu_{2}, \mu_{3}\right) x_{5},
$$

где $\lambda_{1}, \ldots, \lambda_{r}$ - натуральные параметры, $\mu_{1}, \mu_{2}, \mu_{3}$ - переменные для последовательностей натуральных параметров, описъвается ииклическими решениями, если обшее решение описывается преобразованиями

$$
\left\{\begin{array} { l } 
{ x _ { 1 } \rightarrow x _ { 1 } ^ { \alpha } , } \\
{ x _ { 2 } \rightarrow x _ { 1 } ^ { \beta } , } \\
{ x _ { 3 } \rightarrow x _ { 1 } ^ { \gamma } , } \\
{ x _ { 4 } \rightarrow x _ { 1 } ^ { \omega } x _ { 5 } , }
\end{array} \quad \left\{\begin{array}{l}
x_{1} \rightarrow x_{1}^{\alpha}, \\
x_{2} \rightarrow x_{1}^{\beta}, \\
x_{3} \rightarrow x_{1}^{\gamma}, \\
x_{5} \rightarrow x_{1}^{\omega} x_{4},
\end{array}\right.\right.
$$

где $\alpha, \beta, \gamma, \omega$ - натуральные параметры, удовлетворяющие условиям, которые определяются уравнением.

Все уравнения, которые мы рассматриваем в этом параграфе, являются трой-

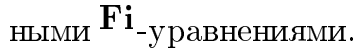

УТВЕРЖДЕНИЕ 89. Общее решение уравнения

$$
x_{3} x_{1} x_{2} x_{1} x_{2} x_{3}^{2}=x_{2} x_{3}^{2} x_{1} x_{3} x_{1} x_{2}
$$

описъввается ииклическими решениями. 
УТВЕРЖДЕНИЕ 90. Общее решение уравнения

$$
x_{3} x_{1} x_{2} x_{1} x_{2} x_{3}^{2}=x_{2} x_{3}^{2} x_{1} x_{2} x_{3} x_{1}
$$

описьвается чиклическими решениями.

УТВЕРЖДЕНИЕ 91. Общее решение уравнения

$$
x_{3} x_{1} x_{2} x_{3} x_{1} x_{2} x_{3}=x_{2} x_{3}^{2} x_{1} x_{2} x_{3} x_{1}
$$

описывается циклическими решениями.

УТВЕРЖДЕНИЕ 92. Общее решение уравнения

$$
x_{3} x_{1} x_{2} x_{3} x_{1} x_{2} x_{3}=x_{2} x_{3} x_{1} x_{2} x_{3}^{2} x_{1}
$$

описывается ииклическими решениями.

УТВЕРЖДЕНИЕ 93. Общее решение уравнения

$$
x_{1} x_{3} x_{2}^{2} x_{1} x_{3}=x_{3} x_{2} x_{1} x_{3} x_{2} x_{1}
$$

описывается циклическими решениями.

УТВЕРЖДЕНИЕ 94. Общее решение уравнения

$$
x_{3} x_{1} x_{2} x_{1} x_{2} x_{3}=x_{2} x_{3} x_{1} x_{2} x_{3} x_{1}
$$

описъввается циклическими решениями.

УТВЕРЖДЕНИЕ 95. Общее решение уравнения

$$
x_{1} x_{3} x_{2}^{2} x_{1} x_{3} P\left(x_{1}, x_{2}, x_{3}\right) x_{4}=x_{3} x_{1} x_{3} x_{2} x_{1} x_{3} x_{2} Q\left(x_{1}, x_{2}, x_{3}\right) x_{5}
$$

описывается циклическими решениями.

ДокАЗАТЕЛЬство следует из утверждения 1.

УТВЕРЖДЕНИЕ 96. Общее решение уравнения

$$
x_{1} x_{3} x_{2}^{2} x_{1} x_{3} P\left(x_{1}, x_{2}, x_{3}\right) x_{4}=x_{3} x_{1} x_{3} x_{2} x_{1}^{2} x_{3} x_{2} Q\left(x_{1}, x_{2}, x_{3}\right) x_{5}
$$

описывается ииклическими решениями.

ДокАЗАТЕЛЬСтво следует из утверждения 1.

УТВЕРЖДЕНИЕ 97. Общее решение уравнения

$$
x_{1} x_{3} x_{2}^{2} x_{1} x_{3} P\left(x_{1}, x_{2}, x_{3}\right) x_{4}=x_{3} x_{2} x_{1} x_{3} x_{1} x_{3} x_{2} Q\left(x_{1}, x_{2}, x_{3}\right) x_{5}
$$

описывается ииклическими решениями.

ДокАЗАТЕЛЬСтво следует из утверждения 4. 
УТВЕРЖДЕНИЕ 98. Общее решение уравнения

$$
x_{1} x_{3} x_{2}^{2} x_{1} x_{3} P\left(x_{1}, x_{2}, x_{3}\right) x_{4}=x_{3} x_{2} x_{1} x_{3} x_{1}^{2} x_{3} x_{2} Q\left(x_{1}, x_{2}, x_{3}\right) x_{5}
$$

описывается циклическими решениями.

ДокАЗАТЕЛЬСтво следует из утверждения 4.

УТВЕРЖДЕНИЕ 99. Общее решение уравнения

$$
x_{3} x_{1} x_{2} x_{3} x_{1} x_{2} x_{3} P\left(x_{1}, x_{2}, x_{3}\right) x_{4}=x_{2} x_{3}^{2} x_{1} x_{2} x_{3}^{2} x_{1} Q\left(x_{1}, x_{2}, x_{3}\right) x_{5}
$$

описъвается циклическими решениями.

ДокАЗАТЕльство следует из утверждения 9.

УТВЕРЖДЕНИЕ 100. Общее решение уравнения

$$
x_{3} x_{1} x_{2} x_{1} x_{2} x_{3} P\left(x_{1}, x_{2}, x_{3}\right) x_{4}=x_{2} x_{3} x_{1} x_{2} x_{3}^{2} x_{1} Q\left(x_{1}, x_{2}, x_{3}\right) x_{5}
$$

описывается ииклическими решениями.

ДокАЗАТЕЛьство следует из утверждения 4.

УТВЕРЖДЕНИЕ 101. Общее решение уравнения

$$
x_{1} x_{3} x_{2}^{2} x_{1} x_{3} P\left(x_{1}, x_{2}, x_{3}\right) x_{4}=x_{3} x_{1}\left(x_{3} x_{2} x_{1}\right)^{\alpha+1} x_{1} x_{3} x_{2} Q\left(x_{1}, x_{2}, x_{3}\right) x_{5},
$$

где $\alpha$ - натуральный параметр, описъвается чиклическими решениями.

ДоКАЗАТЕЛьство следует из утверждений 95, 96.

УТВЕРЖДЕНИЕ 102. Общее решение уравнения

$$
x_{1} x_{3} x_{2}^{2} x_{1} x_{3} P\left(x_{1}, x_{2}, x_{3}\right) x_{4}=x_{3}\left(x_{2} x_{1} x_{3}\right)^{\alpha+1}\left(x_{1} x_{3} x_{2}\right)^{\beta} x_{1}^{2} x_{3} x_{2} Q\left(x_{1}, x_{2}, x_{3}\right) x_{5}
$$

где $\alpha, \beta$ - натуральные параметры, описывается ииклическими решениями.

ДОКАЗАТЕЛЬСтво следует из утверждений 97, 98, 93.

УТВЕРЖДЕНИЕ 103. Общее решение уравнения $x_{1} x_{3} x_{2}\left(x_{2} x_{1}\right)^{\beta+1} x_{1} x_{3} P\left(x_{1}, x_{2}, x_{3}\right) x_{4}=x_{3} x_{1}\left(x_{3} x_{2} x_{1}\right)^{\alpha+1} x_{1} x_{3} x_{2} Q\left(x_{1}, x_{2}, x_{3}\right) x_{5}$,

где $\alpha, \beta$ - натуральные параметры, описывается ииклическими решениями.

ДокАЗАТЕЛЬство следует из утверждений 95, 96.

УТВЕРЖДЕНИЕ 104. Общее решение уравнения

$$
x_{1} x_{3} x_{2}^{2} x_{3} x_{1} P\left(x_{1}, x_{2}, x_{3}\right) x_{4}=\left(x_{3} x_{2}\right)^{\alpha+1} x_{3} x_{1} x_{3} x_{2} Q\left(x_{1}, x_{2}, x_{3}\right) x_{5},
$$

где $\alpha$ - натуральный параметр, описъвается чиклическими решениями.

ДокАЗАТЕЛьСтво следует из утверждений 100-102, 89, 90, 94. 
УТВЕРЖДЕНИЕ 105. Общее решение уравнения

$$
\begin{gathered}
x_{1} x_{3} x_{2}\left(x_{2} x_{1}\right)^{\beta+1}\left(x_{3} x_{2}\right)^{\alpha+1} x_{1} P\left(x_{1}, x_{2}, x_{3}\right) x_{4} \\
=x_{3}\left(x_{3} x_{2}\right)^{\alpha+1} x_{1} x_{3} x_{2} Q\left(x_{1}, x_{2}, x_{3}\right) x_{5},
\end{gathered}
$$

где $\alpha, \beta$ - натуральные параметры, описывается циклическими решениями.

ДОКАЗАТЕЛЬСТво следует из утверждений 103, 104.

УТВЕРЖДЕНИЕ 106. Общее решение уравнения

$$
x_{3} x_{1} x_{2} x_{1}^{\beta+1} x_{3} x_{1} P\left(x_{1}, x_{2}, x_{3}\right) x_{4}=x_{2} x_{3}\left(x_{3} x_{1}\right)^{\alpha+1} x_{2} x_{3} Q\left(x_{1}, x_{2}, x_{3}\right) x_{5},
$$

где $\alpha, \beta$ - натуральные параметры, описьвается ииклическими решениями.

ДокаЗАТЕЛЬство. Пусть преобразование $S$ :

$$
\left\{\begin{array}{l}
x_{1} \rightarrow X_{1} \\
x_{2} \rightarrow X_{2} \\
x_{3} \rightarrow X_{3} \\
\ldots \ldots
\end{array}\right.
$$

где $X_{i}$ - слова в алфавите $(*)$, есть произвольное решение уравнения (106). Индукцией по $\left|X_{1} X_{2} X_{3}\right|$ мы докажем, что преобразование $S$ является циклическим решением.

Уравнение (106) переводится преобразованием

$$
\begin{aligned}
& x_{3} \rightarrow x_{2}^{\gamma} x_{3}, \\
& x_{2} \rightarrow x_{3} x_{2},
\end{aligned}
$$

где $\gamma$-натуральный параметр, в уравнение $E$ :

$$
x_{1} x_{3} x_{2} x_{1}^{\beta+1}\left(x_{3} x_{2}\right)^{\gamma} x_{3} x_{1} \cdots=x_{2} x_{3}\left(\left(x_{3} x_{2}\right)^{\gamma} x_{3} x_{1}\right)^{\alpha+1}\left(x_{3} x_{2}\right)^{\gamma+1} x_{3} \cdots .
$$

Уравнение $E$ с $\partial\left(x_{1}\right) \geqslant \partial\left(x_{2}\right)$ переводится преобразованием $x_{1} \rightarrow x_{2} x_{1}$ в уравнение

$$
x_{1} x_{3} x_{2}\left(x_{2} x_{1}\right)^{\beta+1}\left(x_{3} x_{2}\right)^{\gamma} x_{3} x_{2} x_{1} \cdots=x_{3}\left(\left(x_{3} x_{2}\right)^{\gamma} x_{3} x_{2} x_{1}\right)^{\alpha+1}\left(x_{3} x_{2}\right)^{\gamma+1} x_{3} \cdots,
$$

т.е. в уравнение (105).

Уравнение $E$ с $\partial\left(x_{2}\right) \geqslant \partial\left(x_{1}\right)$ переводится преобразованием $x_{2} \rightarrow x_{1} x_{2}$ в уравнение

$$
x_{3} x_{1} x_{2} x_{1}^{\beta+1}\left(x_{3} x_{1} x_{2}\right)^{\gamma} x_{3} x_{1} \cdots=x_{2} x_{3}\left(\left(x_{3} x_{1} x_{2}\right)^{\gamma} x_{3} x_{1}\right)^{\alpha+1}\left(x_{3} x_{1} x_{2}\right)^{\gamma+1} x_{3} \cdots,
$$

т.е. в уравнение $(106)$. Если $\partial\left(x_{3}\right)=\partial\left(x_{1}\right)=0$, то преобразование $S$ есть циклическое решение. Если $\partial\left(x_{3} x_{1}\right)>0$, то по индуктивному предположению преобразование $S$ должно быть циклическим решением. 
УТВЕРЖДЕНИЕ 107. Общее решение уравнения

$$
x_{1} x_{3} x_{2}^{2} x_{1}^{2} x_{3} P\left(x_{1}, x_{2}, x_{3}\right) x_{4}=x_{3} x_{1}\left(x_{3} x_{2} x_{1}\right)^{\alpha+1} x_{1} x_{3} Q\left(x_{1}, x_{2}, x_{3}\right) x_{5},
$$

әде $\alpha$ - натуральный параметр, описъвается ииклическими решениями.

ДокАЗАТЕЛьство следует из утверждения 1.

УТВЕРЖДЕНИЕ 108. Общее решение уравнения

$$
x_{1} x_{3} x_{2}^{2} x_{1} x_{3} P\left(x_{1}, x_{2}, x_{3}\right) x_{4}=x_{3}\left(x_{2} x_{1} x_{3}\right)^{\alpha+1} x_{1}^{2} x_{3} x_{2} Q\left(x_{1}, x_{2}, x_{3}\right) x_{5},
$$

где $\alpha$ - натуральный параметр, описъвается циклическими решениями.

ДокАЗАТЕльство следует из утверждения 4.

УТВЕРЖДЕНИЕ 109. Общее решение уравнения

$$
\begin{aligned}
& x_{3} x_{1} x_{2} x_{1} x_{2} x_{3}^{2} P\left(x_{1}, x_{2}, x_{3}\right) x_{4} \\
& \quad=x_{2} x_{3}\left(x_{1} x_{2} x_{3}\right)^{\alpha} x_{3}\left(x_{1} x_{2} x_{3}\right)^{\beta} x_{1} x_{3} x_{1} x_{2} Q\left(x_{1}, x_{2}, x_{3}\right) x_{5},
\end{aligned}
$$

где $\alpha, \beta$ - натуральные параметры, описывается ииклическими решениями.

ДокАЗАТЕЛЬСТво следует из утверждений 89, 90.

УТВЕРЖДЕНИЕ 110. Общее решение уравнения

$$
x_{1} x_{3} x_{2}^{2} x_{1} x_{3} P\left(x_{1}, x_{2}, x_{3}\right) x_{4}=x_{3}\left(x_{2} x_{1} x_{3}\right)^{\alpha} x_{1} x_{3} x_{2} x_{1}^{2} x_{3} x_{2} Q\left(x_{1}, x_{2}, x_{3}\right) x_{5},
$$

әде $\alpha$ - натуральный параметр, описывается ииклическими решениями.

ДокАЗАТЕЛьство следует из утверждения 4.

УТВЕРЖДЕНИЕ 111. Общее решение уравнения

$$
x_{1} x_{3} x_{2}^{2} x_{1} x_{3} P\left(x_{1}, x_{2}, x_{3}\right) x_{4}=x_{3}\left(x_{2} x_{1} x_{3}\right)^{\alpha+1} x_{1}^{2} x_{3} x_{2} Q\left(x_{1}, x_{2}, x_{3}\right) x_{5},
$$

әде $\alpha$ - натуральный параметр, описъвается ииклическими решениями.

ДокАЗАТЕЛьСтво следует из утверждения 4.

УТВЕРЖДЕНИЕ 112. Общее решение уравнения

$x_{1} x_{3} x_{2}^{2} x_{3}^{2} x_{1} x_{3} x_{2} x_{3} P\left(x_{1}, x_{2}, x_{3}\right) x_{4}=\left(x_{2} x_{3}\right)^{\alpha+1} x_{3} x_{1} x_{3} x_{2} x_{3} Q\left(x_{1}, x_{2}, x_{3}\right) x_{5}$,

где $\alpha$ - натуральный параметр, описывается ииклическими решениями.

ДокАЗАТЕЛьство следует из утверждений 109-111.

УТВЕРЖДЕНИЕ 113. Общее решение уравнения

$$
\begin{aligned}
& x_{1} x_{3} x_{2}\left(x_{2} x_{3} x_{1} x_{3}\right)^{\alpha+1} x_{2} x_{3} P\left(x_{1}, x_{2}, x_{3}\right) x_{4} \\
& \quad=x_{3}\left(x_{2} x_{3}\right)^{\gamma}\left(x_{2} x_{3} x_{1} x_{3}\right)^{\beta+1} x_{2} x_{3} Q\left(x_{1}, x_{2}, x_{3}\right) x_{5},
\end{aligned}
$$

где $\alpha, \beta, \gamma$ - натуральные параметры, описыввется чиклическими решениямu. 
ДокАЗАТЕЛЬСТво следует из утверждений 108, 112.

УТВЕРЖДЕНИЕ 114. Общее решение уравнения

$$
\begin{aligned}
& x_{1} x_{3} x_{2}\left(x_{2} x_{1} x_{3}\right)^{\alpha+1} x_{2} x_{3} P\left(x_{1}, x_{2}, x_{3}\right) x_{4} \\
& \quad=x_{3}^{2}\left(x_{2} x_{3}\right)^{\gamma}\left(x_{2} x_{1} x_{3}\right)^{\beta+1} x_{2} x_{3} Q\left(x_{1}, x_{2}, x_{3}\right) x_{5},
\end{aligned}
$$

где $\alpha, \beta, \gamma$ - натуральные параметры, описьвается чиклическими решениямu.

ДокАЗАТЕЛЬСТво следует из утверждений 107, 113.

УТВЕРЖДЕНИЕ 115. Общее решение уравнения

$$
\begin{aligned}
& x_{1} x_{3} x_{2}\left(x_{1} x_{3}\right)^{\alpha+1} x_{2} x_{3} P\left(x_{1}, x_{2}, x_{3}\right) x_{4} \\
& \quad=x_{2} x_{3}^{2}\left(x_{2} x_{3}\right)^{\gamma}\left(x_{1} x_{3}\right)^{\beta+1} x_{2} x_{3} Q\left(x_{1}, x_{2}, x_{3}\right) x_{5},
\end{aligned}
$$

где $\alpha, \beta, \gamma$ - натуральные параметры, описьввается ииклическими решениямu.

ДокАЗАТЕльство. Пусть преобразование $S$ :

$$
\left\{\begin{array}{l}
x_{1} \rightarrow X_{1}, \\
x_{2} \rightarrow X_{2}, \\
x_{3} \rightarrow X_{3}, \\
\ldots \ldots \ldots
\end{array}\right.
$$

где $X_{i}$ - слова в алфавите (*), есть произвольное решение уравнения (115). Индукцией по $\left|X_{1} X_{2} X_{3}\right|$ мы докажем, что $S$ - циклическое решение. Уравнение (115) с $\partial\left(x_{1}\right) \geqslant \partial\left(x_{2}\right)$ переводится преобразованием $x_{1} \rightarrow x_{2} x_{1}$ в уравнение

$$
x_{1} x_{3} x_{2}\left(x_{2} x_{1} x_{3}\right)^{\alpha+1} x_{2} x_{3} \cdots=x_{3}^{2}\left(x_{2} x_{3}\right)^{\gamma}\left(x_{2} x_{1} x_{3}\right)^{\beta+1} x_{2} x_{3} \cdots,
$$

т.е. в уравнение (114). Уравнение $(115)$ с $\partial\left(x_{2}\right) \geqslant \partial\left(x_{1}\right)$ переводится преобразованием

$$
\begin{aligned}
& x_{2} \rightarrow x_{1} x_{2}, \\
& x_{3} \rightarrow x_{2}^{\delta} x_{3}, \\
& x_{2} \rightarrow x_{3} x_{2},
\end{aligned}
$$

где $\delta$ - натуральный параметр, в уравнение

$$
\begin{aligned}
& x_{1} x_{3} x_{2}\left(x_{1}\left(x_{3} x_{2}\right)^{\delta} x_{3}\right)^{\alpha+1} x_{1}\left(x_{3} x_{2}\right)^{\delta+1} x_{3} \cdots \\
& \quad=x_{2} x_{3}\left(x_{3} x_{2}\right)^{\delta} x_{3}\left(x_{1}\left(x_{3} x_{2}\right)^{\delta+1} x_{3}\right)^{\gamma}\left(x_{1}\left(x_{3} x_{2}\right)^{\delta} x_{3}\right)^{\beta+1} x_{1}\left(x_{3} x_{2}\right)^{\delta+1} x_{3} \cdots,
\end{aligned}
$$

т.е. в уравнение (115). Если $\partial\left(x_{3}\right)=\partial\left(x_{1}\right)=0$, то $S$ - циклическое преобразование. Если $\partial\left(x_{3} x_{1}\right)>0$, то по индуктивному предположению $S$ должно быть циклическим решением. 
УТВЕРЖДЕНИЕ 116. Общее решение уравнения

$$
\begin{aligned}
& x_{1} x_{3} x_{2} x_{1}^{\beta+1} x_{3} x_{2} P\left(x_{1}, x_{2}, x_{3}, \lambda_{1}, \ldots, \lambda_{r}\right) x_{4} \\
& \quad=x_{2} x_{3}\left(\left(x_{3} x_{2}\right)^{\gamma+1} x_{3}\right)^{\alpha+1} x_{1} Q\left(x_{1}, x_{2}, x_{3}, \lambda_{1}, \ldots, \lambda_{r}\right) x_{5}
\end{aligned}
$$

c $\partial\left(x_{1}\right)<\partial\left(x_{2} x_{3}\left(\left(x_{3} x_{2}\right)^{\gamma+1} x_{3}\right)^{\alpha+1}\right)$, әде $\alpha, \beta, \gamma, \lambda_{1}, \ldots, \lambda_{r}-$ натуральнье параметры, сводится $\kappa\langle 1\rangle,\langle 4\rangle,\langle 67\rangle,\langle 90\rangle,\langle 106\rangle,\langle 115\rangle$.

УТВЕРЖДЕНИЕ 117. Общее решение уравнения

$$
\begin{aligned}
& x_{1} x_{3} x_{2} x_{1}^{\beta+1} x_{3} x_{2} P\left(x_{1}, x_{2}, x_{3}, \lambda_{1}, \ldots, \lambda_{r}\right) x_{4} \\
& \quad=x_{2} x_{3}\left(\left(x_{3} x_{2}\right)^{\gamma+1} x_{3}\right)^{\alpha+1} x_{1} Q\left(x_{1}, x_{2}, x_{3}, \lambda_{1}, \ldots, \lambda_{r}\right) x_{5},
\end{aligned}
$$

где $\alpha, \beta, \gamma, \lambda_{1}, \ldots, \lambda_{r}$ - натуральные параметры, описывается преобразованием

$$
x_{1} \rightarrow\left(x_{2} x_{3}\left(\left(x_{3} x_{2}\right)^{\gamma+1} x_{3}\right)^{\alpha+1}\right)^{\delta} x_{1},
$$

әде $\delta$ - натуральный параметр.

УТВЕРЖДЕНИЕ 118. Общее решение уравнения

$$
\begin{aligned}
& x_{1} x_{3} x_{2} x_{3} x_{2} P\left(x_{1}, x_{2}, x_{3}, \lambda_{1}, \ldots, \lambda_{r}\right) x_{4} \\
& \quad=x_{2} x_{3}\left(\left(x_{3} x_{2}\right)^{\gamma+1} x_{3}\right)^{\alpha+1} x_{1} Q\left(x_{1}, x_{2}, x_{3}, \lambda_{1}, \ldots, \lambda_{r}\right) x_{5}
\end{aligned}
$$

c $\partial\left(x_{1}\right)<\partial\left(x_{2} x_{3}\left(\left(x_{3} x_{2}\right)^{\gamma+1} x_{3}\right)^{\alpha+1}\right)$, где $\alpha, \gamma, \lambda_{1}, \ldots, \lambda_{r}-$ натуральные параметрыл, сводится $\kappa\langle 1\rangle,\langle 4\rangle,\langle 9\rangle,\langle 67\rangle$.

УТВЕРЖДЕНИЕ 119. Общее решение уравнения

$$
\begin{aligned}
& x_{1} x_{3} x_{2} x_{3} x_{2} x_{3} P\left(x_{1}, x_{2}, x_{3}, \lambda_{1}, \ldots, \lambda_{r}\right) x_{4} \\
& \quad=x_{2} x_{3}\left(\left(x_{3} x_{2}\right)^{\gamma+1} x_{3}\right)^{\alpha+1} x_{1} Q\left(x_{1}, x_{2}, x_{3}, \lambda_{1}, \ldots, \lambda_{r}\right) x_{5},
\end{aligned}
$$

где $\alpha, \gamma, \lambda_{1}, \ldots, \lambda_{r}$ - натуральные параметры, описывается преобразованием

$$
x_{1} \rightarrow\left(x_{2} x_{3}\left(\left(x_{3} x_{2}\right)^{\gamma+1} x_{3}\right)^{\alpha+1}\right)^{\beta} x_{1},
$$

$\langle 118\rangle$,

әде $\beta$ - натуральный параметр.

УТВЕРЖДЕНИЕ 120. Общее решение уравнения

$$
x_{3} x_{1} x_{2} x_{1}^{\beta+1} x_{4}=x_{2} x_{3}\left(x_{3} x_{1}\right)^{\gamma+1} x_{2} x_{3} Q\left(x_{1}, x_{2}, x_{3}, \lambda_{1}, \ldots, \lambda_{r}\right) x_{5}
$$

c $\partial\left(x_{3}\right)<\partial\left(x_{2}\right)<\partial\left(x_{3} x_{1}\right)$, әде $\gamma, \beta, \lambda_{1}, \ldots, \lambda_{r}-$ натуральные параметры, описьвается преобразованиями

$$
\begin{array}{ll}
x_{2} \rightarrow x_{3} x_{2}, & x_{2} \rightarrow x_{3} x_{2}, \\
x_{1} \rightarrow x_{2} x_{3} x_{1}, & x_{1} \rightarrow x_{2} x_{1}, \\
\langle 104\rangle ; & x_{3} \rightarrow x_{1} x_{3},
\end{array}
$$


УТВЕРЖДЕНИЕ 121. Общее решение уравнения

$$
\begin{aligned}
& x_{1} x_{3}\left(\left(x_{3} x_{1}\right)^{\gamma+1} x_{3}\right)^{\alpha+1} x_{2} P\left(x_{1}, x_{2}, x_{3}, \lambda_{1}, \ldots, \lambda_{r}\right) x_{4} \\
& \quad=x_{2}\left(\left(x_{3} x_{1}\right)^{\gamma+1} x_{3}\right)^{\beta+1} x_{3} x_{1} Q\left(x_{1}, x_{2}, x_{3}, \lambda_{1}, \ldots, \lambda_{r}\right) x_{5}
\end{aligned}
$$

c $\partial\left(x_{2}\right)<\partial\left(x_{1} x_{3}\left(\left(x_{3} x_{1}\right)^{\gamma+1} x_{3}\right)^{\alpha+1}\right)$, әде $\alpha, \beta, \gamma, \lambda_{1}, \ldots, \lambda_{r}-$ натуральные параметры, сводится $\kappa\langle 67\rangle$.

ДокАЗАТЕЛьство следует из утверждения 9.

УТВЕРЖДЕНИЕ 122. Общее решение уравнения

$x_{1} x_{3}^{\alpha+2} x_{2} P\left(x_{1}, x_{2}, x_{3}, \lambda_{1}, \ldots, \lambda_{r}\right) x_{4}=x_{2} x_{3}^{\beta+2} x_{1} Q\left(x_{1}, x_{2}, x_{3}, \lambda_{1}, \ldots, \lambda_{r}\right) x_{5}$ (122)

c $\partial\left(x_{1}\right)<\partial\left(x_{2} x_{3}^{\beta+2}\right), \partial\left(x_{2}\right)<\partial\left(x_{1} x_{3}^{\alpha+2}\right)$, әде $\alpha, \beta, \lambda_{1}, \ldots, \lambda_{r}-$ натуральные параметры, сводится $\kappa\langle 1\rangle,\langle 67\rangle,\langle 121\rangle$.

УТВЕРЖДЕНИЕ 123. Общее решение уравнения

$$
x_{1} x_{2} x_{3} P\left(x_{1}, x_{2}, x_{3}, \lambda_{1}, \ldots, \lambda_{r}\right) x_{4}=x_{2}^{\alpha+1} x_{1} Q\left(x_{1}, x_{2}, x_{3}, \lambda_{1}, \ldots, \lambda_{r}\right) x_{5},
$$

где $\alpha, \lambda_{1}, \ldots, \lambda_{r}$ - натуральные параметры, описъвается преобразованиeм $\langle 31\rangle,\langle 67\rangle$.

УТВЕРЖДЕНИЕ 124. Общее решение уравнения

$$
x_{2} x_{1} x_{3} P\left(x_{1}, x_{2}, x_{3}, \lambda_{1}, \ldots, \lambda_{r}\right) x_{4}=x_{3} x_{1}\left(x_{3} x_{2} x_{1}\right)^{\alpha+1} Q\left(x_{1}, x_{2}, x_{3}, \lambda_{1}, \ldots, \lambda_{r}\right) x_{5},
$$

где $\alpha, \lambda_{1}, \ldots, \lambda_{r}$ - натуральнье параметры, описывается преобразованиями $\langle 68\rangle ;\langle 72\rangle ;\langle 8\rangle,\langle 67\rangle$ и преобразованием (если $P$ пустое)

$$
x_{4} \rightarrow x_{4} x_{1}\left(x_{3} x_{2} x_{1}\right)^{\alpha} Q x_{5},
$$$$
\langle 60\rangle \text {. }
$$

\section{§5. Двойные $\mathrm{Fi}_{\text {-уравнения }}$}

Произведение параметрических преобразований, состоящее из примитивных параметрических преобразований и не более двух преобразований функцией $\mathbf{F i}$, бу-

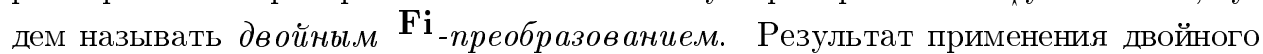
$\mathbf{F i}$-преобразования к равенству слов в алфавите словарных переменных будем на-

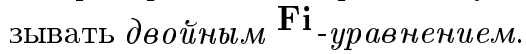

Все уравнения, которые мы рассматриваем в этом параграфе, являются двойньми $\mathbf{F i}$-уравнениями.

УТВЕРЖДЕНИЕ 125. Общее решение уравнения

$$
x_{1} x_{3} x_{2} x_{4}=x_{2} x_{3}^{\alpha+2} x_{1} Q\left(x_{1}, x_{2}, x_{3}, \lambda_{1}, \ldots, \lambda_{r}\right) x_{5},
$$

где $\alpha, \lambda_{1}, \ldots, \lambda_{r}$ - натуральные параметры, описывается преобразованиями

$$
\begin{aligned}
& \left\{\begin{aligned}
x_{1} & \rightarrow{ }^{\mathbf{F i}}\left(x_{1} x_{3}, x_{2} x_{3}^{\alpha+2}\right)^{\mu} x_{1}, \\
x_{2} & \rightarrow \mathbf{F i}_{\left(x_{2} x_{3}^{\alpha+2}, x_{1} x_{3}\right)^{\mu \mid} x_{2},}
\end{aligned}\right. \\
& x_{4} \rightarrow x_{4} Q\left(\mathbf{F i}_{\left.\left(x_{1} x_{3}, x_{2} x_{3}^{\alpha+2}\right)^{\mu} x_{1},{ }^{\mathbf{F i}}\left(x_{2} x_{3}^{\alpha+2}, x_{1} x_{3}\right)^{\mu \mid} x_{2}, x_{3}, \lambda_{1}, \ldots, \lambda_{r}\right) x_{5},}\right.
\end{aligned}
$$

где $\mu$ - переменная для последовательностей натуральных параметров. 
УТВЕРЖДЕНИЕ 126. Общее решение уравнения

$$
x_{1} x_{2} x_{3} P\left(x_{1}, x_{2}, x_{3}, \lambda_{1}, \ldots, \lambda_{r}\right) x_{4}=x_{2} x_{3}^{\alpha+2} x_{1} Q\left(x_{1}, x_{2}, x_{3}, \lambda_{1}, \ldots, \lambda_{r}\right) x_{5},
$$

где $\alpha, \lambda_{1}, \ldots, \lambda_{r}$ - натуральные параметры, описывается преобразованием

$$
\left\{\begin{array}{l}
x_{1} \rightarrow \mathbf{F i}_{\left(x_{1}, x_{2} x_{3}^{\alpha+2}\right)^{\mu} x_{1},} \\
x_{2} \rightarrow \mathbf{F i} \\
\left(x_{2} x_{3}^{\alpha+2}, x_{1}\right)^{\mu \mid} x_{2},
\end{array}\right.
$$

за которым следуют четыре преобразования

$$
\begin{array}{lll}
x_{1} \rightarrow x_{2} x_{3}^{\alpha+1} x_{1}, & x_{1} \rightarrow x_{2} x_{3}^{\beta} x_{1}, & x_{1} \rightarrow x_{2} x_{3}^{\beta} x_{1}, \\
x_{3} \rightarrow x_{1} x_{3}, & x_{3} \rightarrow x_{1} x_{3}, & x_{3} \rightarrow x_{1} x_{3}, \\
\langle 4\rangle, & \langle 87\rangle ; & \langle 88\rangle ; \\
\langle 67\rangle ; & \\
x_{1} \rightarrow x_{2} x_{3}^{\beta} x_{1}, & \\
x_{3} \rightarrow x_{1} x_{3}, & \\
x_{4} \rightarrow x_{4} Q\left(\mathbf{F i}_{\left.\left(x_{1}, x_{2} x_{3}^{\alpha+2}\right)^{\mu} x_{1},{ }^{\mathbf{F i}}\left(x_{2} x_{3}^{\alpha+2}, x_{1}\right)^{\mu \mid} x_{2}, x_{3}\right) x_{5},}\right.
\end{array}
$$

$\langle 60\rangle$,

где $\beta$ - натуральный параметр, $\beta \leqslant \alpha, \quad \mu$ - переменная для последовательностей натуральных параметров.

УТВЕРЖДЕНИЕ 127. Общее решение уравнения

$$
x_{1} x_{3} x_{2} x_{1}^{\beta} x_{i} P\left(x_{1}, x_{2}, x_{3}, \lambda_{1}, \ldots, \lambda_{r}\right) x_{4}=x_{2} x_{3}^{\alpha+2} x_{1} Q\left(x_{1}, x_{2}, x_{3}, \lambda_{1}, \ldots, \lambda_{r}\right) x_{5}
$$

где $i=2,3, \alpha, \beta, \lambda_{1}, \ldots, \lambda_{r}$ - натуральные параметры, описывается преобразованием

$$
\left\{\begin{array}{l}
x_{1} \rightarrow \mathbf{F i}_{\left(x_{1} x_{3}, x_{2} x_{3}^{\alpha+2}\right)^{\mu} x_{1},} \\
x_{2} \rightarrow \mathbf{F i}_{\left(x_{2} x_{3}^{\alpha+2}, x_{1} x_{3}\right)^{\mu \mid} x_{2},}
\end{array}\right.
$$

за которым следуют четыре преобразования

$$
\begin{array}{llll}
x_{1} \rightarrow x_{2} x_{3}^{\alpha+1} x_{1}, & x_{1} \rightarrow x_{2} x_{3}^{\gamma} x_{1}, & x_{2} \rightarrow x_{1} x_{2}, & x_{2} \rightarrow x_{1} x_{2}, \\
x_{3} \rightarrow x_{1} x_{3}, & x_{3} \rightarrow x_{1} x_{3}, & x_{3} \rightarrow x_{2}^{\delta+1} x_{3}, & x_{3} \rightarrow x_{2}^{\delta+1} x_{3}, \\
\langle 4\rangle, & \langle 1\rangle, & x_{2} \rightarrow x_{3} x_{2}, & x_{2} \rightarrow x_{3} x_{2}, \\
\langle 67\rangle ; & \langle 67\rangle ; & \langle 117\rangle ; & \langle 119\rangle,
\end{array}
$$

где $\gamma, \delta$ - натуральные параметры, $\gamma \leqslant \alpha, \mu$ - переменная для последовательностей натуральных параметров.

УТВЕРЖДЕНИЕ 128. Общее решение уравнения

$$
x_{1} x_{2} x_{3} P\left(x_{1}, x_{2}, x_{3}, \lambda_{1}, \ldots, \lambda_{r}\right) x_{4}=x_{2} x_{3}^{\alpha+1} x_{1} Q\left(x_{1}, x_{2}, x_{3}, \lambda_{1}, \ldots, \lambda_{r}\right) x_{5},
$$

где $\alpha, \beta, \lambda_{1}, \ldots, \lambda_{r}$ - натуральнье параметрь, описывается преобразованиямu $\langle 126\rangle ;\langle 4\rangle,\langle 67\rangle$. 
УТВЕРЖДЕНИЕ 129. Общее решение уравнения

$x_{1} x_{2} x_{3} P\left(x_{1}, x_{2}, x_{3}, \lambda_{1}, \ldots, \lambda_{r}\right) x_{4}=x_{3}\left(x_{2} x_{3}\right)^{\alpha+1} x_{1} Q\left(x_{1}, x_{2}, x_{3}, \lambda_{1}, \ldots, \lambda_{r}\right) x_{5}$

где $\alpha, \lambda_{1}, \ldots, \lambda_{r}$ - натуральнье параметры, сводится $\kappa\langle 4\rangle,\langle 67\rangle,\langle 124\rangle,\langle 126\rangle$.

УТВЕРЖДЕНИЕ 130. Общее решение уравнения

$$
\begin{aligned}
& x_{3}\left(x_{1} x_{2}\right)^{2} x_{3} P\left(x_{1}, x_{2}, x_{3}, \lambda_{1}, \ldots, \lambda_{r}\right) x_{4} \\
& \quad=x_{2} x_{3}\left(\left(x_{3} x_{1} x_{2}\right)^{\beta+1} x_{3}\right)^{\alpha+1} x_{1} Q\left(x_{1}, x_{2}, x_{3}, \lambda_{1}, \ldots, \lambda_{r}\right) x_{5},
\end{aligned}
$$

где $\alpha, \beta, \lambda_{1}, \ldots, \lambda_{r}$ - натуральные параметры, описывается преобразованиямu

$$
\begin{array}{ll}
x_{2} \rightarrow x_{3} x_{2}, & x_{3} \rightarrow x_{2}^{\gamma+1} x_{3}, \\
\langle 127\rangle ; & x_{2} \rightarrow x_{3} x_{2},
\end{array}
$$

әде $\gamma$ - натуральный параметр.

УТВЕРЖДЕНИЕ 131. Общее решение уравнения

$$
\begin{gathered}
x_{1} x_{3} x_{2}^{2} x_{3}\left(\left(x_{3} x_{2}\right)^{\gamma+1} x_{3}\right)^{\alpha}\left(x_{3} x_{2}\right)^{\gamma} x_{3} P\left(x_{1}, x_{2}, x_{3}, \lambda_{1}, \ldots, \lambda_{r}\right) x_{4} \\
=x_{2} x_{3}\left(\left(x_{3} x_{2}\right)^{\gamma+1} x_{3}\right)^{\alpha+1} x_{1} Q\left(x_{1}, x_{2}, x_{3}, \lambda_{1}, \ldots, \lambda_{r}\right) x_{5}
\end{gathered}
$$

где $\alpha, \beta, \gamma, \lambda_{1}, \ldots, \lambda_{r}$ - натуральнье параметрыл, сводится $\kappa\langle 1\rangle,\langle 4\rangle,\langle 67\rangle,\langle 130\rangle$.

УТВЕРЖДЕНИЕ 132. Общее решение уравнения

$$
x_{3} x_{1} x_{2} x_{1}^{\beta+1} x_{4}=x_{2} x_{3}\left(x_{3} x_{1}\right)^{\gamma+1} x_{2} x_{3} Q\left(x_{1}, x_{2}, x_{3}, \lambda_{1}, \ldots, \lambda_{r}\right) x_{5},
$$

әде $\gamma, \beta, \lambda_{1}, \ldots, \lambda_{r}$ - натуральные параметры, описывается преобразованием

$$
\left\{\begin{array}{l}
x_{3} \rightarrow \mathbf{F i}_{\left(x_{3} x_{1}, x_{2}\right)^{\mu} x_{3},} \\
x_{2} \rightarrow \mathbf{F i}_{\left(x_{2}, x_{3} x_{1}\right)^{\mu \mid} x_{2},}
\end{array}\right.
$$

где $\mu$ - переменная для последовательностей натуральных параметров.

ДокАЗАТЕЛьство следует из теорем Fi3, Fi4.

УТВЕРЖДЕНИЕ 133. Общее решение уравнения

$$
x_{1} x_{3} x_{2} x_{1}^{\beta+1} x_{4}=x_{2} x_{3}\left(\left(x_{3} x_{2}\right)^{\gamma+1} x_{3}\right)^{\alpha+1} x_{1} Q\left(x_{1}, x_{2}, x_{3}, \lambda_{1}, \ldots, \lambda_{r}\right) x_{5},
$$

где $\alpha, \beta, \gamma, \lambda_{1}, \ldots, \lambda_{r}$ - натуральнье параметрьи, сводится $\kappa\langle 1\rangle,\langle 4\rangle,\langle 67\rangle$, $\langle 130\rangle,\langle 131\rangle,\langle 132\rangle$. 
УТВЕРЖДЕНИЕ 134. Общее решение уравнения

$$
x_{1} x_{3} x_{2} x_{1}^{\beta+1} x_{4}=x_{2} x_{3}^{\alpha+2} x_{1} Q\left(x_{1}, x_{2}, x_{3}, \lambda_{1}, \ldots, \lambda_{r}\right) x_{5}
$$

с $\partial\left(x_{2}\right)<\partial\left(x_{1} x_{3}\right)$, әде $\alpha, \beta, \lambda_{1}, \ldots, \lambda_{r}$ - натуральные параметры, сводится $\kappa\langle 1\rangle,\langle 4\rangle,\langle 67\rangle,\langle 127\rangle,\langle 133\rangle$.

УТВЕРЖДЕНИЕ 135. Общее решение уравнения

$$
x_{1} x_{3} x_{2} x_{1}^{\beta+1} x_{4}=x_{2} x_{3}^{\alpha+2} x_{1} Q\left(x_{1}, x_{2}, x_{3}, \lambda_{1}, \ldots, \lambda_{r}\right) x_{5} \text {, }
$$

әде $\alpha, \beta, \lambda_{1}, \ldots, \lambda_{r}-$ натуральные параметры, описывается преобразованием

$$
x_{2} \rightarrow\left(x_{1} x_{3}\right)^{\gamma} x_{2}
$$

əде $\gamma$ - натуральный параметр.

УТВЕРЖДЕНИЕ 136. Общее решение уравнения

$$
\begin{aligned}
& x_{2} x_{1} x_{3} P\left(x_{1} x_{3} x_{1}, x_{2}, x_{1} x_{3}, \lambda_{1}, \ldots, \lambda_{r}\right) x_{4} \\
& \quad=x_{3} x_{1}\left(\left(x_{1} x_{3}\right)^{\gamma+1} x_{1}\right)^{\alpha+1} x_{2} Q\left(x_{1} x_{3} x_{1}, x_{2}, x_{1} x_{3}, \lambda_{1}, \ldots, \lambda_{r}\right) x_{5}
\end{aligned}
$$

где $\alpha, \gamma, \lambda_{1}, \ldots, \lambda_{r}$ - натуральные параметры, описывается преобразованиямu $\langle 51\rangle ;\langle 133\rangle ;\langle 117\rangle ;\langle 119\rangle$.

УТВЕРЖДЕНИЕ 137. Общее решение уравнения

$$
\begin{aligned}
& x_{1} x_{2} x_{3} P\left(x_{1}, x_{2}, x_{3}, \lambda_{1}, \ldots, \lambda_{r}\right) x_{4} \\
& \quad=x_{2}^{\alpha+1} x_{3} R\left(x_{2}, x_{3}, \lambda_{1}, \ldots, \lambda_{r}\right) x_{1} Q\left(x_{1}, x_{2}, x_{3}, \lambda_{1}, \ldots, \lambda_{r}\right) x_{5}
\end{aligned}
$$

с $\partial\left(x_{2}\right)>\partial\left(x_{1}\right)$, әде $\lambda_{1}, \ldots, \lambda_{r}$ - натуральнье параметрьи, сводится $\kappa\langle 1\rangle,\langle 4\rangle$, $\langle 67\rangle,\langle 126\rangle$.

УТВЕРЖДЕНИЕ 138. Общее решение уравнения

$$
x_{1} x_{3}^{\alpha+2} x_{2} P\left(x_{1}, x_{2}, x_{3}, \lambda_{1}, \ldots, \lambda_{r}\right) x_{4}=x_{2} x_{3}^{\beta+2} x_{1} Q\left(x_{1}, x_{2}, x_{3}, \lambda_{1}, \ldots, \lambda_{r}\right) x_{5},
$$

где $\alpha, \beta, \lambda_{1}, \ldots, \lambda_{r}-$ натуральные параметры, описъввается преобразованием

$$
\left\{\begin{array}{l}
x_{1} \rightarrow \mathbf{F i}_{\left(x_{1} x_{3}^{\alpha+2}, x_{2} x_{3}^{\beta+2}\right)^{\mu} x_{1},} \\
x_{2} \rightarrow \mathbf{F i}_{\left(x_{2} x_{3}^{\beta+2}, x_{1} x_{3}^{\alpha+2}\right)^{\mu \mid} x_{2},}
\end{array}\right.
$$

әде $\mu$ - переменная для последовательностей натуральных параметров.

ДокАЗАТЕЛьство следует из утверждения 35. 
УТВЕРЖДЕНИЕ 139. Общее решение уравнения

$x_{1} x_{3}^{\alpha+1} x_{2} P\left(x_{1}, x_{2}, x_{3}, \lambda_{1}, \ldots, \lambda_{r}\right) x_{4}=x_{2} x_{3}^{\beta+1} x_{1} Q\left(x_{1}, x_{2}, x_{3}, \lambda_{1}, \ldots, \lambda_{r}\right) x_{5},(139)$

где $\alpha, \beta, \lambda_{1}, \ldots, \lambda_{r}$ - натуральные параметры, описьвается преобразованиями $\langle 127\rangle ;\langle 135\rangle ;\langle 125\rangle ;\langle 138\rangle ;\langle 5\rangle ;\langle 67\rangle$.

УТВЕРЖДЕНИЕ 140. Общее решение уравнения

$$
x_{1} x_{2} x_{3} P\left(x_{1}, x_{2}, x_{3}, \lambda_{1}, \ldots, \lambda_{r}\right) x_{4}=x_{3} x_{1}^{\alpha+2} x_{2} Q\left(x_{1}, x_{2}, x_{3}, \lambda_{1}, \ldots, \lambda_{r}\right) x_{5},
$$

где $\alpha, \lambda_{1}, \ldots, \lambda_{r}$ - натуральнье параметрыи, описьввается преобразованиями

$$
\begin{array}{ll}
x_{3} \rightarrow x_{1} x_{3}, & x_{1} \rightarrow x_{3}^{\gamma+1} x_{1}, \\
\langle 139\rangle ; & x_{3} \rightarrow x_{1} x_{3},
\end{array}
$$

где $\gamma$ - натуральный параметр.

УТВЕРЖДЕнИЕ 141. Общее решение уравнения

$$
\begin{aligned}
& x_{1} x_{2} x_{3} P\left(x_{1}, x_{2}, x_{3}, \lambda_{1}, \ldots, \lambda_{r}\right) x_{4} \\
& \quad=x_{3}^{\alpha+1} x_{2} R\left(x_{2}, x_{3}, \lambda_{1}, \ldots, \lambda_{r}\right) x_{1} Q\left(x_{1}, x_{2}, x_{3}, \lambda_{1}, \ldots, \lambda_{r}\right) x_{5},
\end{aligned}
$$

где $\lambda_{1}, \ldots, \lambda_{r}$ - натуральные параметры, описывается преобразованиями

$$
\begin{array}{lll}
\alpha \rightarrow \beta+1, & \alpha \rightarrow 0, & \alpha \rightarrow 0, \\
x_{3} \rightarrow x_{1} x_{3}, & x_{3} \rightarrow x_{1} x_{3}, & x_{3} \rightarrow x_{1} x_{3}, \\
\langle 129\rangle ; & \langle 140\rangle ; & \langle 4\rangle, \\
& & \langle 67\rangle,
\end{array}
$$

где $\beta$ - натуральный параметр.

УТВЕРЖДЕНИЕ 142. Общее решение уравнения

$$
\begin{aligned}
& x_{1} x_{2} x_{3} P\left(x_{1}, x_{2}, x_{3}, \lambda_{1}, \ldots, \lambda_{r}\right) x_{4} \\
& \quad=R\left(x_{2}, x_{3}, \lambda_{1}, \ldots, \lambda_{r}\right) x_{1} Q\left(x_{1}, x_{2}, x_{3}, \lambda_{1}, \ldots, \lambda_{r}\right) x_{5},
\end{aligned}
$$

где $\lambda_{1}, \ldots, \lambda_{r}$ - натуральные параметры, словарные переменные $x_{2}, x_{3}$ входят в $R$, описывается преобразованиями $\langle 137\rangle,\langle 141\rangle$.

УТВЕРЖДЕНИЕ 143. Общее решение уравнения

$$
\begin{aligned}
& x_{1} x_{2} x_{3} P\left(x_{1}, x_{2}, x_{3}, \lambda_{1}, \ldots, \lambda_{r}\right) x_{4} \\
& \quad=x_{3}^{\alpha+1} x_{1} R\left(x_{1}, x_{3}, \lambda_{1}, \ldots, \lambda_{r}\right) x_{2} Q\left(x_{1}, x_{2}, x_{3}, \lambda_{1}, \ldots, \lambda_{r}\right) x_{5},
\end{aligned}
$$

где $\lambda_{1}, \ldots, \lambda_{r}$ - натуральные параметры, сводится $\kappa\langle 142\rangle$. 
УТВЕРЖДЕНИЕ 144. Общее решение уравнения

$$
x_{1} x_{2} x_{3} P\left(x_{1}, x_{2}, x_{3}, \lambda_{1}, \ldots, \lambda_{r}\right) x_{4}=Q\left(x_{1}, x_{2}, x_{3}, \lambda_{1}, \ldots, \lambda_{r}\right) x_{5},
$$

где $\alpha, \lambda_{1}, \ldots, \lambda_{r}$ - натуральные параметры, словарные переменные $x_{1}, x_{2}, x_{3}$ входят в $Q$, описывается преобразованиями $\langle 123\rangle,\langle 143\rangle,\langle 142\rangle$.

УТВЕРЖДЕНИЕ 145. Общее решение уравнения

$$
x_{3} x_{2}^{2} x_{1} x_{3} P\left(x_{1}, x_{2}, x_{3}\right) x_{4}=x_{1}\left(x_{2} x_{1} x_{3}\right)^{\alpha+1} x_{2} Q\left(x_{1}, x_{2}, x_{3}\right) x_{5},
$$

әде $\alpha$ - натуральный параметр, описывается преобразованиями

$$
x_{1} \rightarrow x_{3} x_{1}, \quad x_{3} \rightarrow x_{1} x_{3},
$$

$\langle 144\rangle ; \quad\langle 11\rangle$,

УТВЕРЖДЕНИЕ 146. Общее решение уравнения

$$
x_{2} x_{3} x_{2} x_{1} x_{3} P\left(x_{1}, x_{2}, x_{3}\right) x_{4}=x_{1}\left(x_{1} x_{3}\right)^{\alpha+1} x_{2} Q\left(x_{1}, x_{2}, x_{3}\right) x_{5},
$$

где $\alpha$ - натуральный параметр, описывается преобразованиями

$$
x_{2} \rightarrow x_{1} x_{2}, \quad x_{1} \rightarrow x_{2} x_{1},
$$

$\langle 144\rangle ; \quad\langle 145\rangle$.

УТВЕРЖДЕНИЕ 147. Общее решение уравнения

$$
x_{1} x_{2}^{2} x_{3} P\left(x_{1}, x_{2}, x_{3}\right) x_{4}=x_{3} x_{1} x_{3}^{\alpha} x_{1} x_{2} Q\left(x_{1}, x_{2}, x_{3}\right) x_{5}
$$

с $\partial\left(x_{1}\right)<\partial\left(x_{3}\right)<\partial\left(x_{1} x_{2}^{2}\right)$, где $\alpha$ - натуральный параметр, сводится $\kappa\langle 144\rangle$, $\langle 146\rangle$.

УТВЕРЖДЕНИЕ 148. Общее решение уравнения

$$
\left(x_{3} x_{2}\right)^{\alpha+1} x_{2} x_{1} x_{3} P\left(x_{1}, x_{2}, x_{3}\right) x_{4}=x_{1} x_{2}\left(x_{1} x_{3} x_{2}\right)^{\beta+2} Q\left(x_{1}, x_{2}, x_{3}\right) x_{5},
$$

где $\alpha, \beta$ - натуральные параметры, сводится $\kappa\langle 144\rangle,\langle 11\rangle,\langle 67\rangle$.

УТВЕРЖДЕНИЕ 149. Общее решение уравнения

$$
x_{2}\left(x_{3} x_{2}\right)^{\alpha+1} x_{1} x_{3} P\left(x_{1}, x_{2}, x_{3}\right) x_{4}=x_{1}\left(x_{1} x_{3}\right)^{\beta+2} x_{2} Q\left(x_{1}, x_{2}, x_{3}\right) x_{5},
$$

әде $\alpha, \beta$ - натуральнье параметрьи, описьввется преобразованиями

$$
x_{1} \rightarrow x_{2} x_{1}, \quad x_{2} \rightarrow x_{1} x_{2},
$$




\section{§6. Одинарные ${ }^{\mathrm{Fi}}$-уравнения}

Произведение параметрических преобразований, состоящее из примитивных параметрических преобразований и не более одного преобразования функцией $\mathbf{F i}$, бу-

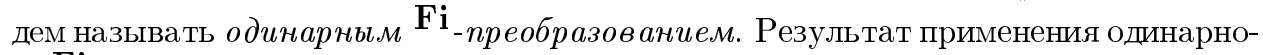

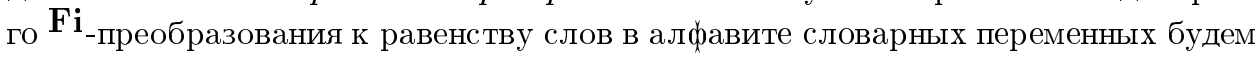

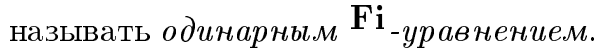

Все уравнения, которые мы рассматриваем в этом параграфе, являются одинарньми $\mathbf{F i}$-уравнениями.

УТВЕРЖДЕНИЕ 150. Общее решение уравнения

$$
x_{1} x_{2}^{2} x_{3} P\left(x_{1}, x_{2}, x_{3}\right) x_{4}=x_{3} x_{1}^{2} x_{2} Q\left(x_{1}, x_{2}, x_{3}\right) x_{5}
$$

описывается преобразованием

$$
\left\{\begin{aligned}
x_{1} & \rightarrow \mathbf{F i}_{\left(x_{1} x_{2}^{2}, x_{3}\right)^{\mu} x_{1},} \\
x_{3} & \rightarrow \mathbf{F i}_{\left(x_{3}, x_{1} x_{2}^{2}\right)^{\mu \mid} x_{3},}
\end{aligned}\right.
$$

$\langle 147\rangle$,

где $\mu$ - переменная для последовательностей натуральных параметров.

ДокаЗАТЕЛЬСтво следует из теорем Fi3, Fi4.

УТВЕРЖДЕнИЕ 151. Общее решение уравнения

$$
x_{2} x_{1} x_{2} x_{3} P\left(x_{1}, x_{2}, x_{3}\right) x_{4}=x_{3} x_{1}^{2} x_{2} Q\left(x_{1}, x_{2}, x_{3}\right) x_{5}
$$

описывается преобразованиями

$$
\begin{array}{ll}
x_{2} \rightarrow x_{3} x_{2}, & x_{3} \rightarrow x_{2} x_{3}, \\
\langle 144\rangle ; & \langle 150\rangle .
\end{array}
$$

УТВЕРЖДЕНИЕ 152. Общее решение уравнения

$$
x_{3} x_{2}\left(x_{2} x_{3}\right)^{\alpha+1} x_{2} x_{1} P\left(x_{1}, x_{2}, x_{3}\right) x_{4}=x_{1}^{2} x_{2} x_{3} Q\left(x_{1}, x_{2}, x_{3}\right) x_{5},
$$

где $\alpha$ - натуральный параметр, описывается преобразованиями

$$
\begin{array}{lll}
x_{3} \rightarrow x_{1} x_{3}, & x_{1} \rightarrow x_{3} x_{2} x_{1}, & x_{1} \rightarrow x_{3} x_{1}, \\
\langle 151\rangle ; & \langle 144\rangle ; & x_{2} \rightarrow x_{1} x_{2},
\end{array}
$$

УТВЕРЖДЕНИЕ 153. Общее решение уравнения

$$
x_{2} x_{1} x_{2} x_{3} P\left(x_{1}, x_{2}, x_{3}\right) x_{4}=x_{3}^{2} x_{1} x_{2} Q\left(x_{1}, x_{2}, x_{3}\right) x_{5}
$$

описьввается преобразованиями

$$
x_{2} \rightarrow x_{3} x_{2}, \quad x_{3} \rightarrow x_{2} x_{3},
$$


УТВЕРЖДЕНИЕ 154. Общее решение уравнения

$$
\left(x_{3} x_{2}\right)^{2} x_{1} P\left(x_{1}, x_{2}, x_{3}\right) x_{4}=x_{2} x_{1}^{2} x_{3} Q\left(x_{1}, x_{2}, x_{3}\right) x_{5}
$$

описывается преобразованием

$$
\begin{aligned}
& x_{2} \rightarrow x_{3}^{\alpha} x_{2}, \\
& x_{3} \rightarrow x_{2} x_{3},
\end{aligned}
$$

әде $\alpha$ - натуральный параметр.

УТВЕРЖДЕНИЕ 155. Общее решение уравнения

$$
x_{3} x_{2}^{2} x_{1} P\left(x_{1}, x_{2}, x_{3}\right) x_{4}=\left(x_{2} x_{1}\right)^{\alpha+1} x_{1} x_{3} Q\left(x_{1}, x_{2}, x_{3}\right) x_{5},
$$

әде $\alpha$ - натуральный параметр, сводится $\kappa\langle 144\rangle,\langle 153\rangle,\langle 154\rangle$.

УТВЕРЖДЕНИЕ 156. Общее решение уравнения

$$
\left(x_{3} x_{2}\right)^{\alpha+1} x_{2} x_{1} P\left(x_{1}, x_{2}, x_{3}\right) x_{4}=x_{1}\left(x_{2} x_{1}\right)^{\beta+1} x_{3} Q\left(x_{1}, x_{2}, x_{3}\right) x_{5},
$$

где $\alpha, \beta$ - натуральнье параметры, описывается преобразованиями

$$
\begin{array}{lll}
x_{1} \rightarrow x_{3} x_{1}, & \alpha \rightarrow 0, & \alpha \rightarrow \gamma+1, \\
\langle 144\rangle ; & x_{3} \rightarrow x_{1} x_{3}, & x_{3} \rightarrow x_{1} x_{3},
\end{array}
$$

әде $\gamma$ - натуральный параметр.

УТВЕРЖДЕНИЕ 157. Общее решение уравнения

$$
x_{2}\left(x_{3} x_{2}\right)^{\alpha+1} x_{1} P\left(x_{1}, x_{2}, x_{3}\right) x_{4}=x_{1}^{\beta+2} x_{3} x_{2} Q\left(x_{1}, x_{2}, x_{3}\right) x_{5},
$$

где $\alpha, \beta$ - натуральнье параметры, описывается преобразованиями

$$
x_{1} \rightarrow x_{2} x_{1}, \quad x_{2} \rightarrow x_{1} x_{2},
$$

УТВЕРЖДЕНИЕ 158. Общее решение уравнения

$$
x_{1} x_{2}^{\alpha+2} x_{3} P\left(x_{1}, x_{2}, x_{3}\right) x_{4}=x_{3} x_{1} x_{3}^{\beta} x_{1} x_{2} Q\left(x_{1}, x_{2}, x_{3}\right) x_{5}
$$

с $\partial\left(x_{1}\right)<\partial\left(x_{3}\right)<\partial\left(x_{1} x_{2}^{\alpha+2}\right)$, әде $\alpha, \beta$ - натуральные параметры, сводится $\kappa\langle 144\rangle,\langle 149\rangle,\langle 157\rangle$. 


\section{$\S 7$. Примитивные параметрические уравнения}

Результат применения примитивного параметрического преобразования к равенству слов в алфавите словарных переменных называется примитивным параметрическим уравнением.

Все уравнения, которые мы рассматриваем в этом параграфе, являются примитивными параметрическими уравнениями.

УТВЕРЖДЕНИЕ 159. Общее решение уравнения

$$
x_{1} x_{2}^{\alpha+2} x_{3} P\left(x_{1}, x_{2}, x_{3}\right) x_{4}=x_{3} x_{1}^{\beta+2} x_{2} Q\left(x_{1}, x_{2}, x_{3}\right) x_{5},
$$

әде $\alpha, \beta$ - натуральные параметры, описывается преобразованием

$$
\left\{\begin{array}{l}
x_{1} \rightarrow \mathbf{F i} \\
\left.x_{3} \rightarrow x_{1} x_{2}^{\alpha+2}, x_{3}\right)^{\mu} x_{1}, \\
\left(x_{3}, x_{1} x_{2}^{\alpha+2}\right)^{\mu \mid} x_{3},
\end{array}\right.
$$

где $\mu$ - переменная для последовательностей натуральных параметров.

ДокАЗАТЕЛьСтво следует из теорем Fi3, Fi4.

УТВЕРЖДЕНИЕ 160. Общее решение уравнения

$$
x_{1}^{\alpha+2} x_{2} x_{3} P\left(x_{1}, x_{2}, x_{3}\right) x_{4}=x_{3} x_{1}^{\beta+2} x_{2} Q\left(x_{1}, x_{2}, x_{3}\right) x_{5},
$$

где $\alpha, \beta$ - натуральные параметры, описывается преобразованиями

$$
\begin{array}{lll}
x_{3} \rightarrow x_{1}^{\gamma} x_{3}, & x_{3} \rightarrow x_{1}^{\alpha+1} x_{3}, & x_{3} \rightarrow x_{1}^{\alpha+2} x_{3}, \\
x_{1} \rightarrow x_{3} x_{1}, & x_{1} \rightarrow x_{3} x_{1}, & \langle 138\rangle, \\
\langle 1\rangle, & \langle 144\rangle ; & \\
\langle 67\rangle ; & &
\end{array}
$$

әде $\gamma$ - натуральный параметр, $\gamma \leqslant \alpha$.

ДокАЗАТЕльство. Уравнение (160) разделяется в список уравнений:

a) (160), $\partial\left(x_{3}\right)<\partial\left(x_{1}^{\alpha+1}\right)$;

b) $(160), \partial\left(x_{1}^{\alpha+1}\right) \leqslant \partial\left(x_{3}\right)<\partial\left(x_{1}^{\alpha+2}\right)$;

c) $(160), \partial\left(x_{1}^{\alpha+2}\right) \leqslant \partial\left(x_{3}\right)$.

УТВЕРЖДЕНИЕ 161. Общее решение уравнения

$$
x_{1} x_{2} x_{1} x_{3} P\left(x_{1}, x_{2}, x_{3}\right) x_{4}=\left(x_{3} x_{2}\right)^{\alpha+1} x_{2} x_{1} Q\left(x_{1}, x_{2}, x_{3}\right) x_{5},
$$

әде $\alpha$ - натуральный параметр, сводится $\kappa\langle 144\rangle,\langle 159\rangle$. 
УТВЕРЖДЕНИЕ 162. Общее решение уравнения

$$
x_{2} x_{1} x_{2} x_{3} P\left(x_{1}, x_{2}, x_{3}\right) x_{4}=x_{3}\left(x_{3} x_{1}\right)^{\alpha+1} x_{2} Q\left(x_{1}, x_{2}, x_{3}\right) x_{5},
$$

әде $\alpha$ - натуральный параметр, описъвается преобразованиями

$$
x_{2} \rightarrow x_{3} x_{2}, \quad x_{3} \rightarrow x_{2} x_{3},
$$

УТВЕРЖДЕНИЕ 163. Общее решение уравнения

$$
\left(x_{1} x_{2}\right)^{2} x_{3} P\left(x_{1}, x_{2}, x_{3}\right) x_{4}=x_{2} x_{3}^{2} x_{1} Q\left(x_{1}, x_{2}, x_{3}\right) x_{5}
$$

сводится $\kappa\langle 161\rangle,\langle 162\rangle$.

УТВЕРЖДЕНИЕ 164. Общее решение уравнения

$$
x_{1} x_{2}^{2} x_{3} P\left(x_{1}, x_{2}, x_{3}\right) x_{4}=\left(x_{2} x_{3}\right)^{\alpha+2} x_{3} x_{1} Q\left(x_{1}, x_{2}, x_{3}\right) x_{5},
$$

әде $\alpha$ - натуральный параметр, сводится $\kappa\langle 144\rangle,\langle 162\rangle,\langle 163\rangle$.

УТВЕРЖДЕНИЕ 165. Общее решение уравнения

$$
x_{1}^{\alpha+2} x_{2} x_{3} P\left(x_{1}, x_{2}, x_{3}\right) x_{4}=x_{3}\left(x_{2} x_{3}\right)^{\beta+1} x_{1} Q\left(x_{1}, x_{2}, x_{3}\right) x_{5},
$$

әде $\alpha, \beta$ - натуральные параметры, описывается преобразованиями

$$
\begin{array}{lll}
x_{3} \rightarrow x_{1} x_{3}, & x_{1} \rightarrow x_{3} x_{2} x_{1}, & x_{1} \rightarrow x_{3} x_{1}, \\
\langle 144\rangle ; & \langle 144\rangle ; & x_{2} \rightarrow x_{1} x_{2},
\end{array}
$$

$\langle 164\rangle$.

УТВЕРЖДЕНИЕ 166. Общее решение уравнения

$$
x_{1} x_{2}^{2} x_{3} P\left(x_{1}, x_{2}, x_{3}\right) x_{4}=x_{3}^{\alpha+1} x_{2} x_{3} x_{1} Q\left(x_{1}, x_{2}, x_{3}\right) x_{5},
$$

где $\alpha$ - натуральный параметр, описывается преобразованиями

$$
\begin{array}{lll}
x_{1} \rightarrow x_{3}^{\beta} x_{1}, & x_{1} \rightarrow x_{3}^{\alpha} x_{1}, & x_{1} \rightarrow x_{3}^{\alpha+1} x_{1}, \\
x_{3} \rightarrow x_{1} x_{3}, & x_{3} \rightarrow x_{1} x_{3}, & \langle 159\rangle, \\
\langle 144\rangle ; & \langle 164\rangle ; &
\end{array}
$$

где $\beta$ - натуральный параметр, $\beta<\alpha$.

УТВЕРЖДЕНИЕ 167. Общее решение уравнения

$$
x_{3}\left(x_{1} x_{3}\right)^{\alpha+1} x_{2} P\left(x_{1}, x_{2}, x_{3}\right) x_{4}=\left(x_{2} x_{1}\right)^{\beta+1} x_{1} x_{3} Q\left(x_{1}, x_{2}, x_{3}\right) x_{5},
$$

где $\alpha, \beta$ - натуральные параметры, сводится $\kappa\langle 144\rangle,\langle 164\rangle$.

УТВЕРЖДЕНИЕ 168. Общее решение уравнения

$$
\left(x_{3}^{\gamma+1} x_{2}\right)^{\alpha+1} x_{2} x_{1} P\left(x_{1}, x_{2}, x_{3}\right) x_{4}=x_{1}\left(x_{2} x_{1}\right)^{\beta+1} x_{3} Q\left(x_{1}, x_{2}, x_{3}\right) x_{5},
$$

әде $\alpha, \beta, \gamma$ - натуральные параметрьи, сводится $\kappa\langle 144\rangle,\langle 167\rangle$. 


\section{§8. Заключительные уравнения}

Все уравнения, которые мы рассматриваем в этом параграфе, являются примитивными параметрическими уравнениями.

УтВеРЖДЕнИЕ 169. Общее решение уравнения

$$
x_{1} x_{2}^{\alpha+2} x_{3} P\left(x_{1}, x_{2}, x_{3}\right) x_{4}=x_{3} x_{1} D\left(x_{3}, x_{3} x_{1}\right) x_{2} Q\left(x_{1}, x_{2}, x_{3}\right) x_{5},
$$

где а - натуральный параметр, D - непустое слово, сводится $\kappa\langle 144\rangle$, $\langle 161\rangle,\langle 166\rangle$.

УТВЕРЖДЕНИЕ 170. Общее решение уравнения

$$
x_{2} x_{1}^{\alpha+1} x_{3} P\left(x_{1}, x_{2}, x_{3}\right) x_{4}=D\left(x_{1}, x_{3}\right) x_{2} Q\left(x_{1}, x_{2}, x_{3}\right) x_{5},
$$

где $\alpha$ - натуральный параметр, $x_{1}, x_{3}$ входят в $D\left(x_{1}, x_{3}\right)$, описъввается преобразованием вида

$$
x_{2} \rightarrow\left(D\left(x_{1} x_{3}\right)\right)^{\lambda} D_{1}\left(x_{1} x_{3}\right) x_{2},
$$

где $\lambda$ - натуральный параметр, $D\left(x_{1} x_{3}\right)=D_{1}\left(x_{1} x_{3}\right) D_{2}\left(x_{1} x_{3}\right)$ для некоторого $D_{2}\left(x_{1} x_{3}\right)$, за которым следуют шесть преобразований:

$\begin{array}{lll}x_{1} \rightarrow x_{2} x_{1}, & x_{1} \rightarrow x_{2} x_{1}, & x_{1} \rightarrow x_{2} x_{1}, \\ \langle 1\rangle, & \langle 144\rangle ; & \langle 169\rangle ; \\ \langle 67\rangle ; & & \\ x_{3} \rightarrow x_{2} x_{3}, & x_{3} \rightarrow x_{2} x_{3}, & x_{3} \rightarrow x_{2} x_{3}, \\ \langle 144\rangle ; & \langle 160\rangle ; & \langle 165\rangle .\end{array}$

УтВеРЖДЕНИЕ 171. Общее решение уравнения

$$
x_{2}\left(x_{3} x_{2}\right)^{\alpha+1} x_{1} P\left(x_{1}, x_{2}, x_{3}\right) x_{4}=D\left(x_{1}, x_{3}\right) x_{2} Q\left(x_{1}, x_{2}, x_{3}\right) x_{5},
$$

где $\alpha$ - натуральный параметр, $x_{1}, x_{3}$ входят в $D\left(x_{1}, x_{3}\right)$, описъвается преобразованием вида

$$
x_{2} \rightarrow\left(D\left(x_{1} x_{3}\right)\right)^{\lambda} D_{1}\left(x_{1} x_{3}\right) x_{2},
$$

где $\lambda$ - натуральный параметр, $D\left(x_{1} x_{3}\right)=D_{1}\left(x_{1} x_{3}\right) D_{2}\left(x_{1} x_{3}\right)$ для некоторого $D_{2}\left(x_{1} x_{3}\right)$, за которым следуют шесть преобразований:

$$
\begin{array}{lll}
x_{2} \rightarrow x_{3}^{\beta} x_{2}, & x_{2} \rightarrow x_{3}^{\beta} x_{2}, & x_{2} \rightarrow x_{3}^{\beta} x_{2}, \\
x_{3} \rightarrow x_{2} x_{3}, & x_{3} \rightarrow x_{2} x_{3}, & x_{3} \rightarrow x_{2} x_{3}, \\
\langle 1\rangle, & \langle 169\rangle ; & \langle 144\rangle ; \\
\langle 67\rangle ; & & \\
x_{2} \rightarrow x_{3}^{\beta} x_{2}, & x_{2} \rightarrow x_{3}^{\beta} x_{2}, & x_{2} \rightarrow x_{3}^{\beta} x_{1} x_{2}, \\
x_{1} \rightarrow x_{2} x_{1}, & x_{1} \rightarrow x_{2} x_{1}, & \langle 170\rangle,
\end{array}
$$

$\langle 168\rangle ; \quad\langle 170\rangle ;$

где $\beta$ - натуральный параметр. 
УТВЕРЖДЕНИЕ 172. Общее решение уравнения

$$
x_{2}^{\alpha+1} x_{1} x_{3} P\left(x_{1}, x_{2}, x_{3}\right) x_{4}=x_{3} D\left(x_{1}, x_{1} x_{3}\right) x_{2} Q\left(x_{1}, x_{2}, x_{3}\right) x_{5},
$$

где $\alpha$ - натуральный параметр, $x_{1}$ входит в $D\left(x_{1}, x_{1} x_{3}\right)$, сводится $\kappa\langle 144\rangle$, $\langle 159\rangle,\langle 171\rangle$.

УТВЕРЖДЕНИЕ 173. Общее решение уравнения

$$
x_{2} C\left(x_{1}, x_{2}\right) x_{3} P\left(x_{1}, x_{2}, x_{3}\right) x_{4}=x_{3} D\left(x_{1}, x_{3}\right) x_{2} Q\left(x_{1}, x_{2}, x_{3}\right) x_{5}
$$

с $\partial\left(x_{3}\right)<\partial\left(x_{2}\right)$, где $x_{1}$ входиткак в $C\left(x_{1}, x_{2}\right)$, так и в $D\left(x_{1}, x_{3}\right)$, описывается преобразованиями

$$
x_{2} \rightarrow x_{3} x_{2}, \quad x_{2} \rightarrow x_{3} x_{2},
$$

УТВЕРЖДЕНИЕ 174. Общее решение уравнения

$$
x_{1} C\left(x_{1}, x_{2}\right) x_{3} P\left(x_{1}, x_{2}, x_{3}\right) x_{4}=x_{3} D\left(x_{1}, x_{3}\right) x_{2} Q\left(x_{1}, x_{2}, x_{3}\right) x_{5}
$$

с $\partial\left(x_{3}\right)<\partial\left(x_{1}\right)$, где $x_{2}$ входит в $C\left(x_{1}, x_{2}\right), x_{1}$ входит в $D\left(x_{1}, x_{3}\right)$, сводится $\kappa\langle 67\rangle,\langle 172\rangle$.

УТВЕРЖДЕНИЕ 175. Параметрическое уравнение

$$
x_{3} P\left(x_{1}, x_{2}, x_{3}\right) x_{4}=D\left(x_{1}, x_{2}\right) x_{3} Q\left(x_{1}, x_{2}, x_{3}\right) x_{5},
$$

где $x_{1}, x_{2}$ входят как в $D\left(x_{1}, x_{2}\right)$, так и в $P\left(x_{1}, x_{2}, x_{3}\right)$, переводится преобразованием вида

$$
x_{3} \rightarrow\left(D\left(x_{1}, x_{2}\right)\right)^{\alpha} D_{1}\left(x_{1}, x_{2}\right) x_{3},
$$

где $\alpha$ - натуральный параметр, $D\left(x_{1}, x_{2}\right)=D_{1}\left(x_{1}, x_{2}\right) D_{2}\left(x_{1}, x_{2}\right)$ для некоторого $D_{2}\left(x_{1}, x_{2}\right)$, в уравнения вида (173), (174).

УТВЕРЖДЕНИЕ 176. Параметрическое уравнение

$$
x_{2}^{\alpha+1} x_{3} P\left(x_{1}, x_{2}, x_{3}\right) x_{4}=D\left(x_{1}, x_{2}\right) x_{3} Q\left(x_{1}, x_{2}, x_{3}\right) x_{5},
$$

әде $\alpha$ - натуральный параметр, $x_{1}, x_{2}$ входят в $D\left(x_{1}, x_{2}\right)$, переводится преобразованиями утверэсдения 30 в уравнение вида (175).

УТВЕРЖДЕНИЕ 177. Параметрическое уравнение

$$
C\left(x_{1}, x_{2}\right) x_{3} P\left(x_{1}, x_{2}, x_{3}\right) x_{4}=D\left(x_{1}, x_{2}\right) x_{3} Q\left(x_{1}, x_{2}, x_{3}\right) x_{5},
$$

где $x_{1}, x_{2}$ входят как в $C\left(x_{1}, x_{2}\right)$, так и в $D\left(x_{1}, x_{2}\right)$, переводится преобразованиями утверәсдения 31 в уравнение вида (67).

УТВЕРЖДЕНИЕ 178. Уравнение вида

$$
P\left(x_{1}, x_{2}, x_{3}\right) x_{4}=Q\left(x_{1}, x_{2}, x_{3}\right) x_{5},
$$

где $x_{1}, x_{2}, x_{3}$ входят как в $P\left(x_{1}, x_{2}, x_{3}\right)$, так и в $Q\left(x_{1}, x_{2}, x_{3}\right)$, распадается в список уравнений вида (175), (176), (177). 


\section{§9. Неполные уравнения}

Все уравнения, которые мы рассматриваем в этом параграфе, являются примитивными параметрическими уравнениями.

УТВЕРЖДЕНИЕ 179. Параметрическое уравнение

$$
P\left(x_{1}, x_{2}\right) x_{3} R\left(x_{1}, x_{2}, x_{3}\right) x_{4}=Q\left(x_{1}, x_{2}\right) x_{5},
$$

әде $x_{1}, x_{2}$ входят как в $P$, так и в $Q$, переводится преобразованием

$$
\left\{\begin{array}{l}
x_{1} \rightarrow x_{1}^{\eta} \\
x_{2} \rightarrow x_{1}^{\theta}
\end{array}\right.
$$

в уравнение (67), где $\eta, \theta$ - натуральные параметры.

УТВЕРЖДЕНИЕ 180. Общее решение уравнения

$$
P\left(x_{1}, x_{2}, x_{3}\right) x_{4}=x_{2} x_{5},
$$

где $x_{2}, x_{3}$ входят в $P$, описъввается преобразованиями вида

$$
\begin{aligned}
& x_{2} \rightarrow\left(R\left(x_{1}, x_{3}\right) S\left(x_{1}, x_{3}\right)\right)^{\alpha} R\left(x_{1}, x_{3}\right) x_{2}, \\
& x_{3} \rightarrow x_{2} x_{3}, \\
& x_{5} \rightarrow Q\left(x_{1}, x_{2}, x_{3}\right) x_{4},
\end{aligned}
$$

где $\alpha$ - натуральный параметр.

УТВЕРЖДЕНИЕ 181. Общее решение уравнения

$$
x_{1} x_{3}^{\alpha+1} x_{2}=x_{2} x_{1} x_{5},
$$

где $\alpha$ - натуральный параметр, описывается преобразованием

$$
\begin{aligned}
& \left\{\begin{aligned}
x_{1} & \rightarrow \mathbf{F i} \\
x_{2} & \rightarrow \mathbf{F i} \\
\left.\left(x_{1} x_{3}, x_{1} x_{3}^{\alpha+1}, x_{2}\right)^{\mu}\right)_{1}, & x_{2},
\end{aligned}\right. \\
& x_{2} \rightarrow x_{1} x_{3}^{\beta} x_{2}, \\
& x_{3} \rightarrow x_{2} x_{3} \text {, }
\end{aligned}
$$

$\langle 180\rangle$,

где $\mu$ - переменная для последовательностей натуральных параметров, $\beta$ натуральный параметр, $\beta \leqslant \alpha$.

УТВЕРЖДЕНИЕ 182. Общее решение уравнения

$$
x_{3}^{\alpha+1} x_{1} x_{2} P\left(x_{1}, x_{2}, x_{3}\right) x_{4}=x_{2} x_{1} x_{5},
$$

где $\alpha$ - натуральный параметр, сводится $\kappa\langle 180\rangle,\langle 181\rangle$. 
УТВЕРЖДЕНИЕ 183. Уравнение вида

$$
P\left(\left(x_{1} x_{2}\right)^{\beta+1} x_{1}, x_{1} x_{2}, x_{3}\right) x_{4}=x_{2} x_{1} x_{5},
$$

где $\beta$ - натуральный параметр $, x_{1}, x_{2}, x_{3}$ входят в $P$, распадается в список уравнений вида (179), (182).

УТВЕРЖДЕНИЕ 184. Общее решение уравнения

$$
P\left(x_{1}, x_{2}, x_{3}\right) x_{4}=x_{2} x_{1} x_{5},
$$

где $x_{1}, x_{2}, x_{3}$ входят в $P$, описьввется преобразованиями

$$
\begin{aligned}
& x_{2} \rightarrow\left(R\left(x_{1}, x_{3}\right) S\left(x_{1}, x_{3}\right)\right)^{\beta} R\left(x_{1}, x_{3}\right) x_{2}, \\
& x_{3} \rightarrow x_{2} x_{3}, \\
& \langle 180\rangle ; \\
& x_{2} \rightarrow\left(R\left(x_{1}, x_{3}\right) S\left(x_{1}, x_{3}\right)\right)^{\beta} R\left(x_{1}, x_{3}\right) x_{2}, \\
& x_{1} \rightarrow x_{2}^{\gamma+1} x_{1}, \\
& x_{2} \rightarrow x_{1} x_{2},
\end{aligned}
$$

$\langle 183\rangle$,

где $\beta, \gamma$ - натуральные параметры.

УТВЕРЖДЕНИЕ 185. Общее решение уравнения

$$
\begin{gathered}
x_{1} P\left(x_{1},\left(\left(x_{3} x_{2}\right)^{\delta+1} x_{3}\right)^{\beta+1} x_{3} x_{2},\left(x_{3} x_{2}\right)^{\delta+1} x_{3}\right) x_{4} \\
=x_{2}\left(x_{3}^{2} x_{2}\right)^{\beta+1}\left(x_{3} x_{2}\left(x_{3}^{2} x_{2}\right)^{\beta+1}\right)^{\alpha} x_{5},
\end{gathered}
$$

где $\alpha, \beta, \delta$ - натуральные параметры, $x_{2}, x_{3}$ входят в $P$, сводится $\kappa\langle 178\rangle$, $\langle 180\rangle,\langle 184\rangle$.

УТВЕРЖДЕНИЕ 186. Общее решение уравнения

$$
x_{1} P\left(x_{1}, x_{3}^{\beta+1} x_{2}, x_{3}\right) x_{4}=x_{2}\left(x_{3}^{\beta+1} x_{2}\right)^{\alpha+1} x_{5},
$$

где $\alpha, \beta$ - натуральные параметры, сводится $\kappa\langle 178\rangle,\langle 180\rangle,\langle 184\rangle$.

УТВЕРЖДЕНИЕ 187. Общее решение уравнения

$$
x_{3}^{\beta+1} x_{1} P\left(x_{1}, x_{3} x_{2}, x_{3}\right) x_{4}=x_{2}\left(x_{3} x_{2}\right)^{\alpha+1} x_{5},
$$

где $\alpha, \beta$ - натуральные параметры, сводится $\kappa\langle 179\rangle,\langle 185\rangle,\langle 186\rangle$.

УТВЕРЖДЕНИЕ 188. Общее решение уравнения

$$
P\left(x_{1}, x_{3} x_{2}, x_{3}\right) x_{4}=x_{2}\left(x_{3} x_{2}\right)^{\alpha+1} x_{5},
$$

где $\alpha$ - натуральный параметр, $x_{1}$ входит в $P$, описъвается преобразования.ми $\langle 179\rangle,\langle 186\rangle,\langle 187\rangle$. 
УТВЕРЖДЕНИЕ 189. Общее решение уравнения

$$
x_{3} P\left(\left(x_{1} x_{2}\right)^{\beta+1} x_{1}, x_{1} x_{2}, x_{3}\right) x_{4}=x_{2} x_{1}\left(\left(x_{1} x_{2}\right)^{\beta+1} x_{1}\right)^{\alpha+1} x_{5},
$$

где $\alpha, \beta$ - натуральные параметры, $x_{1}, x_{2}, x_{3}$ входят в $P$, описывается преобразованием

$$
\begin{aligned}
& x_{3} \rightarrow x_{2} x_{1}\left(\left(x_{1} x_{2}\right)^{\beta+1} x_{1}\right)^{\alpha+1} x_{3}, \\
& x_{5} \rightarrow x_{3} P\left(\left(x_{1} x_{2}\right)^{\beta+1} x_{1}, x_{1} x_{2}, x_{1} x_{2}\left(\left(x_{1} x_{2}\right)^{\beta+1} x_{1}\right)^{\alpha+1} x_{3}\right) x_{4},
\end{aligned}
$$

после чего сводится $\kappa\langle 178\rangle ;\langle 180\rangle ;\langle 184\rangle$.

УТВЕРЖДЕНИЕ 190. Уравнение вида

$$
P\left(\left(x_{1} x_{2}\right)^{\beta+1} x_{1}, x_{1} x_{2}, x_{3}\right) x_{4}=x_{2} x_{1}\left(\left(x_{1} x_{2}\right)^{\beta+1} x_{1}\right)^{\alpha+1} x_{5},
$$

где $\alpha, \beta$ - натуральные параметры, $x_{1}, x_{2}, x_{3}$ входят в $P$, распадается в список уравнений вида (179), (189).

УТВЕРЖДЕНИЕ 191. Общее решение уравнения

$$
x_{2} P\left(x_{1}, x_{2}, x_{3}\right) x_{4}=x_{1}^{\alpha+2} x_{5},
$$

где $\alpha$ - натуральный параметр, $x_{3}$ входит в $P$, описъвается преобразованиями вида

$$
\begin{array}{lll}
x_{2} \rightarrow x_{1}^{\alpha+2} x_{2}, & x_{2} \rightarrow x_{1}^{\alpha+1} x_{2}, & x_{2} \rightarrow x_{1}^{\beta} x_{2}, \\
x_{5} \rightarrow x_{2} P\left(x_{1}, x_{1}^{\alpha+2} x_{2}, x_{3}\right) x_{4} ; & \langle 180\rangle ; & x_{1} \rightarrow x_{2} x_{1},
\end{array}
$$

где $\beta$ - натуральный параметр, $\beta \leqslant \alpha$.

УТВЕРЖДЕНИЕ 192. Общее решение уравнения

$$
P\left(x_{1}, x_{2}, x_{3}\right) x_{4}=x_{2} x_{1}^{\alpha+2} x_{5},
$$

где $\alpha$ - натуральный параметр $, x_{1}, x_{2}, x_{3}$ входят в $P$, описывается преобразованиями вида

$$
\begin{aligned}
& x_{2} \rightarrow\left(R\left(x_{1}, x_{3}\right) S\left(x_{1}, x_{3}\right)\right)^{\beta} R\left(x_{1}, x_{3}\right) x_{2}, \\
& x_{3} \rightarrow x_{2} x_{3}, \\
& \langle 191\rangle ; \\
& x_{2} \rightarrow\left(R\left(x_{1}, x_{3}\right) S\left(x_{1}, x_{3}\right)\right)^{\beta} R\left(x_{1}, x_{3}\right) x_{2}, \\
& x_{1} \rightarrow x_{2}^{\gamma+1} x_{1}, \\
& x_{2} \rightarrow x_{1} x_{2},
\end{aligned}
$$

әде $\beta, \gamma-$ натуральные параметры. 
УТВЕРЖДЕНИЕ 193. Параметрическое уравнение

$$
x_{3} P\left(x_{1}, x_{2}, x_{3}\right) x_{4}=Q\left(x_{1}, x_{2}\right) x_{5},
$$

әде $x_{1}, x_{2}$ входят как в $P$, так и в $Q$, переводится преобразованием

$$
\begin{aligned}
& x_{3} \rightarrow Q\left(x_{1}, x_{2}\right) x_{3}, \\
& x_{5} \rightarrow x_{3} P\left(x_{1}, x_{2}, Q\left(x_{1}, x_{2}\right) x_{3}\right) x_{4}
\end{aligned}
$$

и преобразованием вида

$$
\begin{aligned}
x_{3} & \rightarrow R\left(x_{1}, x_{2}\right) x_{3}, \\
x_{2} & \rightarrow x_{3} x_{2}
\end{aligned}
$$

к уравнениям вида (192), (188), (180), (184), (178).

УТВЕРЖДЕНИЕ 194. Параметрическое уравнение

$$
x_{1}^{\alpha+1} x_{3} P\left(x_{1}, x_{2}, x_{3}\right) x_{4}=x_{2}^{\beta+1} x_{1} Q\left(x_{1}, x_{2}\right) x_{5},
$$

где $\alpha, \beta$ - натуральные параметры, $x_{2}$ входит в $P$, переводится преобразованием $\langle 65\rangle \kappa$ уравнениям вида (193), (67).

УТВЕРЖДЕНИЕ 195. Уравнение вида

$$
P\left(x_{1}, x_{2}, x_{3}\right) x_{4}=Q\left(x_{1}, x_{2}\right) x_{5}
$$

где $x_{1}, x_{2}, x_{3}$ входят в $P, x_{1}, x_{2}$ входят в $Q$, распадается в список уравнений вида (179), (194), (193).

УТВЕРЖДЕНИЕ 196. Общее решение уравнения

$$
P\left(x_{1}, x_{2}, x_{3}\right) x_{4}=x_{5}
$$

описывается преобразованием

$$
x_{5} \rightarrow P\left(x_{1}, x_{2}, x_{3}\right) x_{4} .
$$

УТВЕРЖДЕНИЕ 197. Уравнение вида

$$
P\left(x_{1}, x_{2}, x_{3}\right) x_{4}=Q\left(x_{1}, x_{2}, x_{3}\right) x_{5},
$$

где $x_{1}, x_{2}, x_{3}$ входят в $P$, распадается в список уравнений вида (178), (195), (180), (191), (196). 
УТВЕРЖДЕНИЕ 198. Общее решение уравнения

$$
x_{1} x_{2}^{\alpha+1} x_{4}=x_{3}^{\beta+1} x_{1} Q\left(x_{3} x_{1}, x_{3}\right) x_{5},
$$

где $\alpha, \beta$ - натуральные параметры, описьвается преобразованиями

$$
\begin{array}{ll}
\alpha \rightarrow 0, & \alpha \rightarrow \delta+1, \\
x_{1} \rightarrow x_{3}^{\gamma} x_{1}, & x_{1} \rightarrow x_{3}^{\gamma} x_{1}, \\
x_{3} \rightarrow x_{1} x_{3}, & x_{3} \rightarrow x_{1} x_{3},
\end{array}
$$

$\langle 180\rangle ; \quad\langle 191\rangle$,

где $\gamma, \delta$ - натуральные параметры.

УТВЕРЖДЕНИЕ 199. Общее решение уравнения

$$
x_{2}\left(x_{3} x_{2}\right)^{\alpha+1} x_{4}=\left(x_{1} x_{3}\right)^{\beta+2} x_{5},
$$

где $\alpha, \beta$ - натуральные параметры, описывается преобразованиями

$$
\begin{array}{ll}
x_{2} \rightarrow x_{1} x_{2}, & x_{1} \rightarrow x_{2} x_{1}, \\
\langle 197\rangle ; & \langle 197\rangle .
\end{array}
$$

УТВЕРЖДЕНИЕ 200. Общее решение уравнения

$$
P\left(x_{1}, x_{2}\right) x_{4}=x_{3} x_{5}
$$

описьвается преобразованиями вида

$$
\begin{aligned}
& x_{3} \rightarrow P\left(x_{1}, x_{2}\right) x_{3}, \quad x_{3} \rightarrow R\left(x_{1}, x_{2}\right) x_{3}, \quad x_{3} \rightarrow R\left(x_{1}, x_{2}\right) x_{3}, \\
& x_{4} \rightarrow x_{3} x_{5} ; \quad x_{1} \rightarrow x_{3} x_{1}, \quad x_{2} \rightarrow x_{3} x_{2}, \\
& x_{5} \rightarrow x_{1} Q\left(x_{3} x_{1}, x_{2}\right) x_{4} ; \quad x_{5} \rightarrow x_{2} Q\left(x_{3} x_{2}, x_{1}\right) x_{4} .
\end{aligned}
$$

УТВЕРЖДЕНИЕ 201. Общее решение уравнения

$$
x_{1} x_{2} x_{4}=x_{3}^{\beta} x_{5},
$$

где $\beta$ - натуральный параметр, сводится $к\langle 29\rangle,\langle 200\rangle$.

УТВЕРЖДЕНИЕ 202. Общее решение уравнения

$$
x_{2}\left(x_{3} x_{2}\right)^{\alpha+1} x_{4}=x_{1} x_{3} x_{5},
$$

где $\alpha$ - натуральный параметр, сводится $к\langle 29\rangle,\langle 197\rangle,\langle 200\rangle$.

УТВЕРЖДЕНИЕ 203. Общее решение уравнения

$$
x_{2}^{\alpha+2} x_{4}=x_{3}\left(x_{1} x_{3}\right)^{\beta+1} x_{5},
$$

где $\alpha, \beta$ - натуральные параметры, сводится $\kappa\langle 197\rangle,\langle 199\rangle,\langle 202\rangle$. 
УТВЕРЖДЕНИЕ 204. Общее решение уравнения

$$
x_{2} x_{1}\left(\left(x_{1} x_{2}\right)^{\gamma+1} x_{1}\right)^{\beta+1} x_{4}=x_{3}^{\alpha+2} x_{5},
$$

где $\alpha, \beta, \gamma-$ натуральнье параметры, сводится $\kappa\langle 197\rangle$.

УТВЕРЖДЕНИЕ 205. Общее решение уравнения

$$
\left(x_{2} x_{1}\right)^{\alpha+1} x_{4}=x_{1} x_{3}^{\beta} x_{5}
$$

где $\alpha, \beta$ - натуральные параметры, сводится $\kappa\langle 200\rangle,\langle 201\rangle,\langle 204\rangle$.

УТВЕРЖДЕНИЕ 206. Общее решение уравнения

$$
x_{2} x_{1}^{\alpha+1} x_{4}=Q\left(x_{1}, x_{3}\right) x_{5},
$$

әде $\alpha$ - натуральный параметр, сводится $\kappa\langle 67\rangle,\langle 197\rangle,\langle 203\rangle,\langle 205\rangle$.

УТВЕРЖДЕНИЕ 207. Общее решение уравнения

$$
x_{2} P\left(x_{1}, x_{2}\right) x_{4}=x_{3} Q\left(x_{1}, x_{3}\right) x_{5},
$$

әде $x_{1}$ входит в $P$, сводится $\kappa\langle 197\rangle,\langle 206\rangle$.

УТВЕРЖДЕНИЕ 208. Общее решение уравнения

$$
x_{2} P\left(x_{1}, x_{2}\right) x_{4}=x_{1}^{\alpha+1} x_{3} Q\left(x_{1}, x_{3}\right) x_{5},
$$

әде $x_{1}$ входит в $P$, сводится $\kappa\langle 197\rangle,\langle 198\rangle,\langle 207\rangle$.

УТВЕРЖДЕНИЕ 209. Уравнение вида

$$
x_{2} P\left(x_{1}, x_{2}\right) x_{4}=Q\left(x_{1}, x_{3}\right) x_{5}
$$

где $x_{1}$ входит в $P, x_{3}$ входит в $Q$, распадается в список уравнений ви$\partial a(208),(207)$.

УТВЕРЖДЕНИЕ 210. Общее решение уравнения

$$
x_{1} x_{2}^{\alpha+2} x_{4}=x_{3}^{\beta} x_{5}
$$

где $\alpha, \beta$ - натуральные параметры, сводится $\kappa\langle 29\rangle,\langle 203\rangle$.

УТВЕРЖДЕНИЕ 211. Общее решение уравнения

$$
x_{1}^{\alpha+1} x_{2} P\left(x_{1}, x_{2}\right) x_{4}=x_{3} Q\left(x_{1}, x_{3}\right) x_{5}
$$

сводится $\kappa\langle 197\rangle,\langle 198\rangle,\langle 201\rangle,\langle 210\rangle$.

УТВЕРЖДЕНИЕ 212. Уравнение вида

$$
P\left(x_{1}, x_{2}\right) x_{4}=Q\left(x_{1}, x_{3}\right) x_{5},
$$

где $x_{1}, x_{2}$ входят в $P, x_{3}$ входит в $Q$, распадается в список уравнений вида (211), (209).

УТВЕРЖДЕНИЕ 213. Уравнение вида

$$
P\left(x_{1}, x_{2}, x_{3}\right) x_{4}=Q\left(x_{1}, x_{2}, x_{3}\right) x_{5}
$$

распадается в список уравнений вида (197), (67), (212), (211), (196). 


\section{Список литературы}

1. Хмелевский Ю.И. Решение уравнений в словах с тремя неизвестньми // Докл. АН CCCP. 1967. Т. 177. С. 1023-1025.

2. Хмелевский Ю. И. Уравнения в свободной полугруппе // Труды МИАН. 1971. Т. 107.

3. Makanin G.S. On general solution of equations in a free semigroup // Word equations and related topics. 2nd International Workshop. Rouen, France, 1991. Berlin: Springer-Verlag, 1993. P. 1-5. (Lecture Notes in Comput. Sci. V. 677.)

4. Makanin G.S., Abdulrab H., Maksimenko M. Formal parametric equations // Lecture Notes in Comput. Sci. 1995. V. 965. P. 353-362.

5. Makanin G. S. Multiplication of natural number parameters and equations in a free semigroup // Trans. Amer. Math. Soc. 1996. V. 348. № 12. P. 4813-4824.

6. Makanin G.S., Abdulrab H., Goralcik P. Functions for the general solution of parametric word equations // Logical foundations of computer science. 4th International Symposium, Yaroslavl, Russia, 1997. Berlin: Springer-Verlag, 1997. P. 189-202. (Lecture Notes in Comput. Sci. V. 1234.)

7. Makanin G.S., Makanina T.A. Functions for parametrization of solutions of an equation in a free monoid // Trans. Amer. Math. Soc. 2000. V. 352. № 1. P. 1-54.

8. Маканин Г. С., Маканина Т. А. Параметризация решений некоторых уравнений квадратов в свободном моноиде // Дискрет. матем. 1999. Т. 11. № 3. С. 133-148.

9. Makanin G.S., Makanina T.A. Parametrization of solutions of parametric equation in free monoid // Theoret. Comput. Sci. 2000. V. 242. P. 403-475.

10. Маканин Г. С. Параметризация решений уравнения $x^{-1} y^{-1} x y z^{-1} v^{-1} z v=1$ в свободной группе // Дискрет. матем. 2001. Т. 13. № 2. С. 35-88.

11. Маканин Г. С., Савушкина А.Г. Уравнение в свободной группе, определяющее крашеные косы // Матем. заметки. 2001. Т. 70. № 4. С. 591-602.

12. Маканин Г. С. Конечная параметризация решений уравнений в свободном моноиде. I // Матем. сб. 2004. Т. 195. № 2. С. 41-90. 